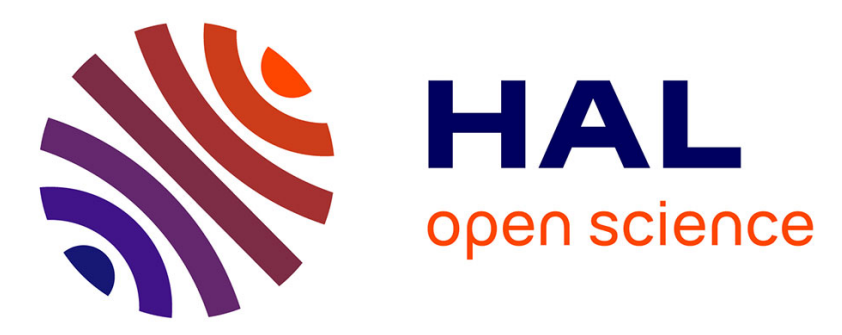

\title{
Age-related breeding success in little penguins: a result of selection and ontogenetic changes in foraging and phenology
}

Claire Saraux, André Chiaradia

\section{To cite this version:}

Claire Saraux, André Chiaradia. Age-related breeding success in little penguins: a result of selection and ontogenetic changes in foraging and phenology. Ecological monographs, 2021. hal-03322572

\section{HAL Id: hal-03322572 \\ https://hal.science/hal-03322572}

Submitted on 19 Aug 2021

HAL is a multi-disciplinary open access archive for the deposit and dissemination of scientific research documents, whether they are published or not. The documents may come from teaching and research institutions in France or abroad, or from public or private research centers.
L'archive ouverte pluridisciplinaire HAL, est destinée au dépôt et à la diffusion de documents scientifiques de niveau recherche, publiés ou non, émanant des établissements d'enseignement et de recherche français ou étrangers, des laboratoires publics ou privés. 
Published in Ecological Monographs

1 Running head: age-dependent breeding performances

2

3

4

5

6

$7 \quad{ }^{1}$ IPHC, DEPE, CNRS-Université de Strasbourg, UMR7178, 23 rue du Loess, 67037 Strasbourg

$8{ }^{2}$ Conservation Department Phillip Island Nature Parks, PO Box 97, Cowes, VIC 3922, Australia

$9 \quad *$ Corresponding Author: Claire Saraux, claire.saraux@iphc.cnrs.fr

12 Open Research statement: Data are available from the Dryad Digital Repository at the 13 following address: https://doi.org/10.5061/dryad.m37pvmd2h. 


\section{Abstract}

Reproductive performance typically improves with age, reaching a plateau at middle age and subsequently declining in older age classes (senescing individuals). Three potential nonexclusive mechanisms can explain the improvement in reproductive performance with age: (1) selection (poor quality individuals are removed from the population with increasing age), (2) constraint (individual efficiency increases through experience) and (3) restraint (reproductive investment increases with age as the residual reproductive value decreases). While all three mechanisms received strong empirical support, few studies have aimed at teasing apart those hypotheses and understanding their underlying functioning. In little penguins (Eudyptula minor), we used a 19-year longitudinal dataset on breeding and foraging of more than 450 individuals to investigate the effect of age on breeding success. We separated within- from among-individual age-effects using state-of-the-art statistical methods (within-subject centering and population change decomposition). We then assessed whether within-individual changes in breeding resulted from ontogenetic changes in foraging performances, breeding phenology or access to mates and nest sites. Fidelity and assortative pairing explained the high correlation in male and female ages within a pair. Breeding performances followed a typical bell-shaped curve with performance increasing up to 8 years-old, before reaching a plateau and subsequently declining after age 16. Both selection and within-individual processes occurred, although within-individual changes dominated differences in age-dependent breeding success. The selective appearance had almost no effect (apart from ages 2 to 3 ), and selective disappearance mostly affected changes at old ages (above 16), although they were also responsible for the slight increase in reproductive performances from ages 5 to 8 . Focusing on within-individual changes, birds exhibited higher performances at middle ages, with birds foraging better, laying earlier and changing partner and nest less often. Their reproductive investment did not vary with age for females and slightly decreased for males. This supports the constraint hypothesis but not the restraint one. Finally, the 
39 increase in breeding performances at young ages was explained by the age-related increase in 40 foraging performances during chick-rearing and advancement of laying. In contrast, reproductive 41 senescence was defined by a general decrease in bird performances.

42 Keywords: assortative pairing, constraint, experience, restraint, seabirds, selective 43 disappearance, senescence 
In vertebrates, a population's reproductive performance typically improves with age until it reaches a plateau at middle ages (Clutton-Brock 1988, Forslund and Pärt 1995, Kirkwood and Austad 2000, Jones et al. 2014). How reproductive performance varies with age in animals closer to the end of life is more debated. There is a traditional view that breeding success decreases with age due to senescence (Nussey et al. 2013). However, there is growing evidence that terminal investment could also occur due to fewer future breeding prospects, resulting in very high terminal reproductive performance (Clutton-Brock 1984, Duffield et al. 2017). Three hypotheses have been proposed to explain the age-related change in reproductive performance (Forslund and Pärt 1995): (1) the selection hypothesis; the progressive disappearance of lowquality phenotypes in the population (Vaupel and Yashin 1985), (2) the constraint hypothesis; gained experience results in an increased individual breeding performance with age (Curio 1983) and (3) the restraint hypothesis; reproductive effort and allocation increase with age according to life-history trade-offs as future breeding prospects decrease (Williams 1966). These three hypotheses are not mutually exclusive, and it is often challenging to distinguish them separately (Forslund and Pärt 1995). The selection hypothesis attributes differences to interindividual intrinsic characteristics present at or developed soon after birth, also known as fixed heterogeneity (Wilson and Nussey 2010, Bergeron et al. 2011). In more detail, within a population, different phenotypes might cause specific individuals to have higher reproductive quality than others (Vaupel and Yashin 1985). If individuals of varying quality also have intrinsically different survival probabilities, i.e. lower quality individuals having a lower chance of survival (Nur 1988), the apparent increase in reproductive performance with age in a population might result from the progressive disappearance of poorer quality phenotypes in older 
phenotypic disappearance of lower capability individuals (Mauck et al. 2004, Reid et al. 2008, McCleery et al. 2008, Lescroël et al. 2009, Rebke et al. 2010).

On the other hand, the constraint and restraint hypotheses result from within-individual changes over time, also known as dynamic-heterogeneity (Tuljapurkar et al. 2009). The constraint hypothesis (Curio 1983) states that the lack of accumulated skills limits younger individuals' success (Balbontin et al. 2007). As individuals age, they gain experience and become more competent at providing for their offspring (Part 1995). Therefore, increased experience allows greater parental output per unit cost. There is widespread support for the effect of experience on performance, particularly in birds (e.g. Hatch and Westneat, 2007), mammals (Broussard et al. 2003, Bowen et al. 2006) and reptiles (Schwartz et al. 2007). Still, the mechanisms through which breeding performance increases remain unclear. Several parameters are known to affect reproductive performances. For instance, access to mates (or mates of better quality) might be reserved to more territorial individuals, a characteristic that often varies with age (see for instance, species with dominance-rank-based mating tactics, e.g. (Coltman et al. 2002, Spong et al. 2008)). Similarly, age can affect an individual's access to a higher quality breeding territory (Ferrer and Bisson 2003), which in return has important consequences on reproductive success (Catchpole and Phillips 1992, Siikamäki 1995). Breeding phenology also affects breeding success, primarily due to the match of the energetically costly reproductive period with prey abundance (Durant et al. 2007, Reed et al. 2013). Indeed, reproduction is an energetically expensive event, and the acquisition of food and energy is thus expected to be one of the most important drivers of breeding performance (Lescroël et al. 2010). Thus, previous studies have proposed that the peak in middle-age breeding performances could result from improved foraging performances at those ages (Cunningham et al. 2017). Foraging performance results from the balance between foraging efficiency, i.e. the rate of energy gain, and foraging effort, i.e. the total amount of energy spent foraging (Daunt et al. 2007). As such, this performance may 
94 be improved by individuals learning more efficient hunting techniques minimizing energy expenditure and time spent hunting for prey (Marchetti and Price 1989, Daunt et al. 2007). This in turn may increase the dietary range available by providing older individuals with access to better foraging territories (Pärt 2001) and more nutritionally valuable prey items (Rutz et al. 2006, Le Vaillant et al. 2013). Therefore, accumulated parental experience allows adults to provide more for their chicks without increasing the associated costs, resulting in parents becoming more competent foragers with age (Curio 1983, Galbraith et al. 1999). A decrease in foraging performance associated with other aspects of senescence could also explain patterns of decrease in breeding success towards the end of an individual's life (Nussey et al. 2008, Reed et al. 2008, Rebke et al. 2010).

While foraging performances represent the energy available for an individual, the next question is to assess how individuals allocate this energy to either self-maintaining their body condition or reproducing (Levins 1968, Stearns 1989). The restraint hypothesis explains the change in resource allocation with age between adults and their offspring due to the change in residual reproductive value with age (Williams 1966). Residual reproductive value is defined as the average future reproductive success for individuals of that age and sex in the population (Williams 1966). Young long-lived individuals are predicted to favour their survival over the current breeding attempt so they preferentially allocate resources to the maintenance of body condition rather than to reproduction (Drent and Daan 1980), as they have a high residual reproductive value. In theory, with increasing age and fewer breeding attempts left, energy should then be preferentially allocated to reproduction and chick provisioning rather than selfmaintenance so that reproductive effort increases with age (Williams 1966, Trivers 1972, Pianka and Parker 1975). However, only a few studies have shown an age-related increase in the reproductive effort using proxies such as territory defence or breeding frequency (Descamps et al. 2007). Thus, rather than a continuous increase in breeding effort, another possible 
consequence of the restraint hypothesis would be a sudden increase in breeding effort past a certain age or ageing threshold, when the residual reproductive value would subsequently become very small (Duffield et al. 2017). Such an increase observed in several species is often named terminal investment (Clutton-Brock 1984, Part et al. 1992, Velando et al. 2006, Froy et al. 2013).

Although age-specific reproductive success changes have been shown in a large number of studies, the three hypotheses are usually investigated separately (but see Mauck, Huntington, \& Doherty Jr, 2012; Orgeret, Weimerskirch, \& Bost, 2016). Longitudinal data studies are required to separate within- and among-individual effects and reveal the underlying processes leading to population-level changes (van de Pol and Verhulst 2006, Nussey et al. 2008, Rebke et al. 2010). Further, methods to distinguish between constraint (reproductive quality) and restraint (reproductive effort) need to be developed to understand better the ontogenetic changes occurring within individuals.

Seabirds' long life spans and slow ageing rates for their body size make them ideal models to study the effect of age on life-history traits (Holmes and Austad 1995). Furthermore, seabirds' highly stochastic environments place strong selection on coping with an unpredictable food supply, perhaps enhancing individual differences in performance capabilities (Saraux et al. 2011a). The average lifespan of a little penguin, Eudyptula minor, is estimated to be 6.5 years (Reilly and Cullen 1979), but this is due to a highly skewed age-specific survival probability (substantial juvenile mortality of $83 \%$ within the first year; Sidhu, Catchpole, \& Dann, 2007). Conversely, adult mortality is rather low (17\%; (Sidhu et al. 2007)) and the oldest little penguin found to date was 25 years old (Dann et al. 2005). Although this might appear relatively young for a seabird, it would still relate as long-lived for a vertebrate. Therefore, a large part of the population is made up of old birds that could significantly influence population dynamics. Further, previous studies on the little penguin have shown age-dependent foraging performances 
144 (Zimmer et al. 2011), age-segregation in foraging areas (Pelletier et al. 2014) and an increase in 145 breeding performances in the first years (Nisbet and Dann 2009). Individual foraging 146 performance is crucial in little penguins, as they have one of the shortest known foraging ranges 147 of seabirds (<30km: Collins, Cullen, \& Dann, 1999) and are limited by the need to return to land 148 to feed their chicks. Thus, we expected the constraint hypotheses to play an essential role in increasing breeding performances with age. Yet, little penguins show a trade-off between chick provisioning and body maintenance, exhibiting a bimodal pattern of foraging trip duration (Saraux et al. 2011b) similar to other seabirds (Chaurand and Weimerskirch 1994, RopertCoudert et al. 2004, Kato et al. 2008). This behaviour suggests that parents might modulate their effort and thus show potential for the restraint hypothesis. Finally, we showed previously that some individuals performed consistently better by making more foraging trips than others to feed the chicks, which could be related to their intrinsic quality (Saraux et al. 2011a). If such intrinsic quality is linked to reproduction and survival, it might lead to selective disappearance that affects population performance.

In this study, we investigated the effect of age on breeding performances of little penguins at Phillip Island using an automatic monitoring system from 2001 to 2018 on more than 450 birds. We proposed a framework to test for all 3 hypotheses: constraint, restraint and selection. First, we examined the age-dependence in breeding performance and, more specifically, its improvement from early life onwards, the decrease late in life due to senescence processes or the increase at the very end due to terminal investment. Second, using mixed modelling (van de Pol and Verhulst 2006, Fay et al. 2020) and decomposition of population change in a trait (Rebke et al. 2010), we distinguished between within- and among-individual variability in breeding performances. Third, we constructed performance and investment indices to separate between

167 the restraint and constraint hypotheses. Finally, we focused on ontogenetic changes in 168 reproduction (i.e. within individual age-related changes) and analyzed whether these were 
related to improved foraging performances, breeding timing, or access to better partners or breeding territories.

\section{Materials and methods}

\section{Little penguin monitoring}

In this study, the little penguin breeding colony is located at Summerland Peninsula on the western tip of Phillip Island $\left(33^{\circ} 31^{\prime} \mathrm{S}, 145^{\circ} 09^{\prime} \mathrm{E}\right)$, Victoria, Australia. This megacolony consists of approximately 32,000 little penguin breeding adults (Sutherland and Dann 2014). All penguins included in this study nested in 100 artificial burrows (nest boxes) established behind the Penguin Parade® area with an annual occupation between 43 and 77 boxes (Chiaradia and Kerry 1999). This study covers 19 breeding seasons from 2000 to 2018 (inclusive), where each breading season spans from August to March the year after (the austral summer). The penguin population on Phillip Island has been monitored since 1967 (Reilly and Cullen 1979, 1981, Dann and Cullen 1990). Between 1968 and 2000, individuals were marked and identified by flipper bands, and since 2000, all monitored birds have been implanted with subcutaneous passive transponder tags (Allflex, Capalaba, Australia) between the scapulae (shoulder blades; Chiaradia \& Kerry, 1999). This intensive monitoring of the population means that the colony consists primarily of known-age individuals, with most penguins marked as chicks. Sex was determined by bill measurements when the birds were first found in the colony as adults (Arnould et al. 2004). The artificial nest boxes were monitored three times a week during the breeding season to assess breeding phenology and success of each pair. Individuals with transponders were detected in the nest using a purpose-built handheld transponder reader (Kean Electronics, Australia). This monitoring ensured a recapture probability close to one and informed us about laying date, the number of clutches per season ( 1 to 3$)$, as well as the number of fledglings per breeding event ( 0 
to 2). Chicks were considered fledged when they had all their adult feathers and were older than 40 days when last seen. Fledging success was estimated as the number of chicks that survived to fledging per breeding event. Based on this monitoring, breeding was also divided in three consecutive phases: i) incubation of the eggs, ii) guard of the chicks, a period when chicks are always guarded by one of the two parents and iii) post-guard, when both parents are out at sea foraging during the day and only return a few hours at night (but not always every night) to feed the chicks.

\section{Foraging variables}

Attendance data were recorded by an Automatic Penguin Monitoring System (APMS) located along the main path into the Penguin Parade study site, and were used to calculate three foraging parameters: foraging trip duration, change in adult body mass, and chick meal size (Kerry et al. 1993, Chiaradia and Kerry 1999, Saraux et al. 2011a). Note that the APMS records started in June 2001, and foraging variables are thus absent in the 2000 season. The APMS consists of an electronic transponder reader and a weighing platform that records the transponder identification, mass, and time of a penguin crossing the weighbridge (Robinson et al. 2005). APMS is calibrated weekly to account for tare drifts, and deviance is considered for weight assessment (Salton et al. 2015). However, automatic weighing can sometimes provide inconsistent data due to multiple birds crossing at once. Only individual masses ranging between 700 and $1700 \mathrm{~g}$ were considered, based on field observation of body mass range (Salton et al. 2015). Only penguins with a breeding record (i.e. for which at least one egg was laid) were included in the foraging dataset so that changes in foraging performance could be directly related to reproductive success $\left(\mathrm{N}_{\text {penguins }}=465\right)$.

Foraging trip duration was calculated as the number of days at sea between colony visits. Foraging duration was measured in days, as little penguins depart before sunrise and return after sunset (Chiaradia and Kerry 1999, Daniel et al. 2007). Only trips that lasted between 1 and 16 
days were considered for incubation and post-guard, and trips up to 3 days in guard (longer trips were classed as false negatives or abandonment and end of breeding; (Chiaradia and Kerry 1999, Kato et al. 2008, Saraux et al. 2011b)). Short trips were defined as those lasting less than or equal to 2 days ( $\leq 2$ days) whereas long trips lasted more than 2 days ( $\geq 3$ days; (Saraux et al. 2011b))

Two mass parameters were determined for each trip: parental body mass change and chick meal size (Saraux et al. 2011a). Adult body mass change was the amount of mass change per foraging trip and was calculated as the difference in body mass between when a penguin goes out and when it returns from the foraging trip. Based on the manual weighing of individuals in the colony, changes in body mass outside of the 0 to $600 \mathrm{~g}$ range during chick-rearing (guard and post guard) and -75 to $500 \mathrm{~g}$ range during incubation were considered inconsistent and discarded.

Chick meal size was the amount of food given to the chick(s) and was assumed to be the mass difference between the penguin arriving at the colony after the foraging trip and returning once again to sea. Chick meal size could solely be estimated during post-guard, when little penguins only spend a few hours in the colony (Daniel et al. 2007), the mass loss while in the colony assumed to be due to feeding their chicks rather than their metabolism. Only chick meal sizes smaller than $550 \mathrm{~g}$ were included in data analysis following previous direct observations (Chiaradia and Nisbet 2006).

\section{Performance and investment indices}

To distinguish between the restraint and constraint hypotheses, we built five indices related to performance or investment. First, performances were evaluated through 4 indices all translating a different aspect: i) phenology based on a bird's laying date, ii) propensity to keep the same nest, iii) propensity to keep the same partner and iv) foraging performances. The foraging performance index was based on a bird's mass gain during its foraging trip. Because body mass change varied according to breeding stage and foraging trip duration, we applied a correction to 
this parameter. During incubation, mass change increased with trip duration (LMM: $t=19.291$, $\mathrm{P}<0.001, \mathrm{n}=3849, \mathrm{~N}=278$; Appendix S1: Fig. $\mathrm{S} 1$ ), so that we used the residuals of this regression as an index of foraging performances during incubation. During guard, as mass change was not related to trip duration $(\mathrm{LMM}: \mathrm{t}=-1.493, \mathrm{P}=0.135, \mathrm{n}=7000, \mathrm{~N}=263$, Appendix S1: Fig. S1), we used body mass change directly to indicate foraging performance. Finally, during post-guard, mass change was better explained by the short/long trip binary variable than by the actual foraging trip duration $(\triangle \mathrm{AIC}=-31$; Appendix S1: Fig. S1). Therefore, we used the model's residual, which explained mass change by the binary short-long trip variable (LMM: $\mathrm{t}=8.599, \mathrm{P}<0.001, \mathrm{n}=15656, \mathrm{~N}=247)$ as an index of foraging performance during post-guard.

To quantify parental investment into chicks, we needed to account for the trade-off between reproduction and maintenance and use the proportion of energy that is allocated to reproduction (represented here by chick meal size) compared to the total amount of energy (assessed here by the change in adult body mass during a foraging trip) (Viblanc et al. 2016). Because chick meal size is likely to depend on the birds' performance, we corrected this by the body mass gained during a given trip. To estimate the total investment over the breeding event rather than the immediate investment associated to each trip, we averaged chick meal size and the birds' performance over the entire breeding event. These data were only available over the post-guard stage, which is the only time we have access to chick meal size. Thus, the investment index was estimated as the residual of the regression of average chick meal size on average body mass change (see Fig. 1). Positive residuals indicate a higher investment towards reproduction than the average population, while negative ones represent a lower investment towards reproduction than the average, i.e., a higher investment towards soma than the average population investment towards soma. 
268 All statistical analyses were carried out in the R 3.5.0 statistical program (R Development Core 269 Team, 2018). Variables were considered significant at $\mathrm{P}<0.05$. All results are represented as mean \pm standard error.

\section{Removing inter-annual variations: year centering}

272 Because environmental fluctuations can generate significant year-to-year variations in little penguin phenology and breeding success, we worked on relative indices rather than absolute ones. Relative indices indicate how an individual behaves compared to the average population behaviour in that year. To do so, we centered (i.e. substracted the annual means to) all our variables (as suggested in Rebke et al., 2010). This procedure removed year-to-year variation and removed any potential bias linked to covariation between age and year (e.g. very old birds usually only present at the end of datasets). All analyses were then performed on relative fledging success, relative laying dates, relative probability to change nest or partner, relative investment and relative foraging performances (for which the year-centering was applied per breeding stage). For instance, a positive relative laying date indicated that a bird reproduced later than the average population in a given year.

\section{Age-dependent breeding performances}

As age effects are usually non-linear (bell-shaped or reaching a plateau), we first described ageeffects on relative fledging success (number of chicks fledged per breeding event relative to the mean of the year) using generalized additive mixed models (GAMM). Because breeding success is the same for the male and female of a pair, data were not independent and we ran separate analyses for both sexes. The penguins' identification number was entered as a random effect in all models, to account for the repeated recordings of the same individual in the longitudinal dataset. 
To separate the selection hypothesis from the constraint or restraint hypotheses, we applied two different approaches. First, based on an adaptation of the within-individual centering method (van de Pol and Wright 2009) and quadratic effects (Fay et al. 2020), we separated within- and among-individual age-related changes in breeding performances. Briefly, within-individual centering was performed by subtracting an individual's mean age from its age in different years.

The centered age and the mean age were then used as independent variables representing within and among-individual variation, respectively. Only individuals known during their entire lifetime were included, i.e.individuals that had not been seen at the colony after 2016, corresponding to 301 penguins, and not including those 172 penguins that were still alive at the end of the study period. The same procedure was conducted using the squared age variable (age ${ }^{2}$ - mean (age 2$)$ ) (more details in Fay et al., 2020). Again, this model was run separately on males and females. The model thus read:

$$
N b \text { of fledglings } s_{r e l} \sim a g e_{c e n t e r e d}+a g e_{c e n t e r e d}^{2}+\text { meanage }+ \text { mean }\left(a g e^{2}\right)+(1 \mid I D)
$$

A stepwise backward model selection was applied to this model until the AIC stopped decreasing. Note that the same model including higher-order linearity of age (i.e. cubic effects or $\mathrm{age}^{4}$ ) displayed higher AIC (not presented here), so that quadratic effects seemed to better explain changes in relative number of fledglings.

Second, we used a decomposition method (Rebke et al. 2010) that focuses on change in a given variable from one age to the next. It decomposes the change into 1) those that occurred within individuals and 2) changes occurring as new individuals appear in the breeding population and old ones disappear. The method is based on the fact that the change in a parameter at the population scale is the sum of three processes, which can be expressed by the following equation:

$$
P_{a+1}-P_{a}=\left(P_{a+1}-p_{a+1}\right)+\left(p_{a+1}-p_{a}\right)+\left(p_{a}-P_{a}\right)
$$


Where $\mathrm{P}_{\mathrm{a}}$ is the average of a trait at the population scale at age $\mathrm{a}, \mathrm{p}_{\mathrm{a}}$ the average trait at age a of individuals that were present at both ages a and $a+1$. The first term represents the difference between the population average at age $a+1$ and the average at a+1 over birds that were already present at age a (selective appearance). The second term is the difference between age a+1 and age a for birds that were present at both ages (within-individual ontogenetic changes) and the last term the difference between the average at age a over birds that will also be present at age a+1 and the population average at age a (selective disappearance). Because this method investigates changes from one age to the next, we averaged relative fledgling success over the breeding season rather than a breeding event (i.e average over all clutches). Age-related changes in relative fledging success were thus decomposed following this method and age classes were then regrouped according to their similarities in population trends and the importance of the 3 different processes. Again, this method was applied only on a subset of the data comprising just penguins that were monitored during their entire lifetime, i.e. that died over the study period.

\section{Constraint and restraint hypothesis: Age-dependent changes in performances and investment}

To test the constraint and restraint hypotheses, we first investigated age-effects on the 4 performance indices (foraging performances, laying date, access to better breeding territory or partners) as well as the reproductive investment index described above using generalized additive mixed models. Note that foraging performances were averaged over a breeding event (or over breeding stages) rather than keep it at the trip scale to avoid individuals for which more trips were monitored to be overrepresented and bias the results. Because laying date and divorce are the same for the male and female of a pair (if an individual divorces, its partner does as well by definition), we ran separate models for males and females for these two parameters. For the other three parameters, sex was included as a covariate in the model as well as an interaction of sex and age, to test whether age effects differed between the sexes, while the penguins' identification number was entered as a random effect to account for the repeated recordings of 
the same individual in the longitudinal dataset. Second, for those parameters that did vary with age, we investigated within- versus among-individual age-related changes in the performance and investment parameters using Rebke's decomposition method including only individuals known all their life. Once again separate analyses were run for males and females for laying date, divorce rates and those parameters for which the sex*age interaction was significant in the GAMM. Relative foraging parameters as well as relative propension to change nest or divorce were averaged over the breeding season rather than a breeding event (i.e. over all clutches) in this analysis as it investigates changes from one age to the next. However, only first clutches were considered to estimate the laying date of the breeding season so that a bird that laid more than one clutch would not be considered as late because of that. Because this analysis was done to explain changes in breeding performances, results are presented grouped per the same age class defined by the decomposition of relative fledging success.

\section{Influence of parameters to explain ontogenetic changes in breeding performances}

Finally, to understand ontogenetic changes in breeding success, we used linear mixed models (LMM) for each age class where important ontogenetic changes had been observed in breeding success to link relative fledging success with relative foraging performances, laying date, access to better breeding territory or partners and investment. Here, again, two separate analyses were run: one for males and one for females, while the individual's identity was added as a random factor in each model to account for repeated measurements. Whenever the individual explained a trivial amount of variance, it was removed and a linear model was run instead to avoid problem of linear mixed model singularity and convergence.

Similarly, we used linear models to investigate the link between ontogenetic changes in relative fledging success with ontogenetic changes in these 5 parameters (foraging performances, laying date, nest or partner changes, and reproductive investment). Ontogenetic changes were assessed by estimating the slopes of linear relationships of age on the studied parameter for each 
individual that was present in the given age class. Because sample sizes varied strongly between the studied parameters, models were run with a unique explanative variable each time so as to maximize statistical power.

367

\section{$\underline{\text { Results }}$}

A total of 465 known-age individuals and 2752 breeding records (note that this corresponds to 1376 breeding events, as one breeding event is associated with 2 parents: the male and female) were recorded between 2000 and 2018. Fledging success varied between 0 and 2 fledged chicks per breeding event (average $0.99 \pm 0.02$ ). It varied strongly among years (range: $0.31 \pm 0.05$ in $2018-1.56 \pm 0.07$ in 2002), justifying the year centering we used for further analyses. The ages of breeding individuals varied between 2 and 21 years. Male and female ages were positively related (Fig. 2A; LM: $\mathrm{t}=24.159, \mathrm{P}<0.001, \mathrm{R}^{2}=0.30, \mathrm{n}=1348$ breeding events). Little penguins had a low divorce rate (27\% of cases where the previous partner was still alive). This means that birds remained with the same partner in $70 \%$ of the cases (the remaining $3 \%$ corresponding to widowers), a high fidelity that could explain the positive relationship between male and female age, as individuals of a pair would grow old together. Yet, focusing on breeding events where birds had to choose a partner (i.e. first recorded breeding events or new partner), male and female ages were still positively related (Fig. $2 B ; L M: t=9.945, P<0.001, R^{2}=0.17, n$ $=479$ ), suggesting some assortative pairing. Further, birds changed burrows from one breeding event to another $46 \%$ of the time. Laying in this species is relatively asynchronous and varies from year to year, as shown by the wide range of laying dates in this study from the $19^{\text {th }}$ of May to the $16^{\text {th }}$ of January, with a peak in mid-October (annual means: $16^{\text {th }}$ of August $-21^{\text {st }}$ of November). 
Finally, a total of 42,473 trips was monitored, but because the mass was not always recorded,

387

388

389

390

391

392

393

394

395

396

397

398

399

400

401

402

403

404

405

406

407 foraging performances were known for only 26,705 trips (15\% in incubation, $26 \%$ in guard and $59 \%$ in post-guard).

\section{Age-dependent breeding performances}

The relative number of chicks fledged (per breeding event) was significantly affected by age in both males and females (GAMMor: $\mathrm{F}=11.51, \mathrm{P}<0.001, \mathrm{n}=1346, \mathrm{~N}=229, \mathrm{R}^{2}=0.03$ and $\Delta \mathrm{AIC}=-29$ with the null model, GAMMO $: \mathrm{F}=11.53, \mathrm{P}<0.001, \mathrm{n}=1348, \mathrm{~N}=232, \mathrm{R}^{2}=0.03$ and $\triangle \mathrm{AIC}=-30$ with the null model, Fig.3A). Briefly, it strongly increased from age 2 to 8 (predicted values from $-0.38 \pm 0.08$ in females and $-0.36 \pm 0.08$ in males to $0.15 \pm 0.04$ in both males and females) before levelling-off. It remained above 0 until age 13 before decreasing quite strongly $(-0.44 \pm 0.23$ for females and $-0.41 \pm 0.22$ in males at age 21$)$. Similarly, the relative number of clutches per season varied significantly with age in both males and females (GAMM ơ: $\mathrm{F}=7.06, \mathrm{P}<0.001, \mathrm{n}=1093, \mathrm{~N}=229, \mathrm{R}^{2}=0.02, \Delta \mathrm{AIC}=-12$ with the null model. GAMMO $: \mathrm{F}=3.63, \mathrm{P}=0.010, \mathrm{n}=1095, \mathrm{~N}=232, \mathrm{R}^{2}=0.01 \Delta \mathrm{AIC}=-2$ with the null model, Fig. $\left.3 \mathrm{~B}\right)$. The relative number of clutches increased until age 8 before decreasing again in males, while the increase in young females was slower but lasted until age 11, before decreasing again, a decrease occurring at a faster pace after 15 (Fig. 3B).

\section{Selection hypothesis: Within- or among-individual differences}

Using the within-subject centering for a linear and quadratic age effect showed that the age effect observed on breeding performance resulted from both within- and among-individual effects in both males and females (see Table 1). Selection by AIC retained all 4 variables (mean age, centered age and their squares) in females, while mean age ${ }^{2}$ was not selected in males. A 
quadratic effect of age was observed within individual in both males and females, showing an increase of breeding performance with age before decreasing again (Fig. 4). The peak in reproductive performances changed depending on the mean age of an individual from after midlife for individuals that disappear young (centered age $=4$ ) to before mid-life for individuals of high longevity (centered age $=-2$ ), suggesting a common age of maximal performances around age 9-10 for both males and females. Further, individuals with a lower mean age (i.e. that disappeared from the population earlier), had a lower breeding performance (Fig. 4). This was translated in the model by a quadratic effect of mean individual age in females and a positive linear effect in males (i.e. among-individual variation due to selective disappearance). Breeding performances at a given age increased with individual mean age (i.e., longevity), although this increase was more important at lower mean age than at higher ones, where it levelled off for females (Fig. 4). Finally, within-individual age effects explained more deviance in breeding performances than among-individual age effects $(16.23 v$ s. 5.4 , i.e. 75 vs. $25 \%$ of the age effects respectively in males and 20.06 vs. 15.17 , i.e. $57 \%$ vs. $43 \%$ in females).

Second, we applied a decomposition of age changes that revealed both ontogenetic changes and population changes. Age-dependent breeding performances were explained mainly by ontogenetic changes (median of the proportion of population changes explained by ontogenetic changes $=102 \%$ and $107 \%$ for males and females respectively vs. $2 \%$ and $3 \%$ for selective disappearance and $0 \%$ for selective appearance; Fig. 5A\&C). The selective appearance had a negative effect on relative reproduction changes between age 2 and 3 in both males and females (though more strongly in females) but almost no impact afterwards (Fig. 5A\&C, Appendix S1: Fig. S2). In other words, individuals breeding for the first time at age 3 had a lower breeding success at age 3 than individuals that had already bred the previous year. Further, breeding performances increased due to ontogenetic development until age 5 in females and age 6 in males and decreased after age 16 due to ontogenetic senescence. Finally, selective disappearance 
had a small positive effect on breeding performances at the population level, i.e. individuals that died earlier had lower breeding performances, an effect mainly occurring between age 5 and 8 or after age 16 in both males and females (Fig. 5A\&C, Appendix S1: Fig. S2). Thus, we defined 4 age categories: i) from ages 2 to 5, when breeding performances increased at the population level (0.24 and 0.40 fledgling per age year in males and females respectively) primarily due to ontogenetic development despite the selective appearance of lower breeding quality individuals at age 3, ii) from ages 5 to 8 , when breeding performances continued to increase in males (although 2.5 times less than in the first age category) and remained stable in females at the population level due to selective disappearance, while within-individual performances had levelled-off or started to slightly decrease, iii) from ages 8 to 16 when breeding performances were stable (very slight decrease) at the population level, and iv) from ages 16 to 20, when breeding performances decreased at the population level (-0.13 and -0.28 per age year in males and females respectively) due to ontogenetic changes but despite selective disappearance of lower breeding quality individuals (Fig. 5C \& D).

\section{Constraint hypothesis: Age-dependent changes in performance}

We studied the constraint hypothesis through age-dependent changes in 4 different indicators of performance (Fig. 6). First, foraging performances at the population level were significantly affected by age, albeit slightly differently in males and females (GAMM: $F=15.58, P<0.001$ for the interaction age*sex and $\mathrm{F}=11.36, \mathrm{P}<0.001$ for age $\mathrm{n}=3462, \mathrm{~N}=288 ; \mathrm{R}^{2}=0.02$, Fig. 6A). For both sexes, foraging performances increased until age 10 before decreasing until age 20. While the increase in early ages was slightly stronger in females, the decrease at old ages was stronger in males (Fig. 6A). Note that similar trends were observed when looking at foraging performances during guard or post-guard separately but not during incubation, when 456 foraging performances did not vary consistently with age (Appendix S1: Fig. S3 A, B \& C). 

22.05, $\mathrm{P}<0.001, \mathrm{n}=1071, \mathrm{~N}=227 ; \mathrm{R}^{2}=0.10, \Delta \mathrm{AIC}=-92$ with the null model; GAMMO $: \mathrm{F}=$ 19.24, $\mathrm{P}<0.001, \mathrm{n}=1067, \mathrm{~N}=231, \mathrm{R}^{2}=0.09, \Delta \mathrm{AIC}=-81$ with the null model). Briefly, it decreased until age 6 or 7 for females and males respectively (age-7 males laid eggs on average 35 days earlier than age- 2 males and age-6 females 33 days earlier than age- 2 females), remained relatively stable until age 14 or 16 in females and males before increasing, older birds laying $~ 20$ days later than intermediate birds (Fig. 6B). Further, the proportion of birds that changed nests decreased with age (GAMM: $\mathrm{F}=6.57, \mathrm{P}=0.003, \mathrm{n}=1692, \mathrm{~N}=333, \mathrm{R}^{2}=0.01$, $\Delta \mathrm{AIC}=-6$ with the null model, Fig. $6 \mathrm{C})$, regardless of sex $(\mathrm{F}=1.18, \mathrm{P}=0.308)$, with a rapid decrease from 3 to 8 . From age 8 onwards, the proportion of birds changing nests did not show any trend, although the variability increased at old ages (Fig. 6C). Finally, the propensity of birds to divorce was significantly affected by age in males (GAMM ơ: $\mathrm{F}=8.23, \mathrm{P}<0.001, \mathrm{n}=$ $715, \mathrm{~N}=156, \mathrm{R}^{2}=0.03, \Delta \mathrm{AIC}=-12$ with the null model) and tended to be affected by age in females (GAMM $\mathrm{O}: \mathrm{F}=3.03, \mathrm{P}=0.033, \mathrm{n}=718, \mathrm{~N}=157, \mathrm{R}^{2}=0.01, \Delta \mathrm{AIC}=0$ with the null model). Birds decreased their divorce rates from ages 3 to 9, especially males (Fig. 6D). From age 9 onwards, the proportion of males changing partners increased while the proportion of females changing partners was stable until age 16 before it slightly increased again at older ages (Fig. 6D).

Because these changes occurred at the population level and could be unrelated to withinindividual changes, we decomposed age changes according to ontogenetic changes, selective appearance and disappearance (Fig. 6E-H). We found that age changes in foraging performances, laying date and the probability of changing nest burrow were primarily due to within-individual ontogenetic changes. However, females appearing later in the breeding population tended to lay their eggs later and exhibit high foraging performances (Fig. $6 \mathrm{E \& F}$ ). 
The importance of ontogenetic changes compared to selective appearance or disappearance was also observed when looking at foraging performances in guard or post-guard (Appendix S1: Fig. S3 D, E \& F). Age changes in the probability to change partners resulted from a combination of selective disappearance and ontogenetic changes in males (Fig 6H): from ages 2 to 5 the important decrease in divorce rates was due to the disappearance of birds divorcing, while from ages 5 to 8 , it was due to individuals divorcing less. Finally, the increase of divorce rates after age 16 was due to individuals increasing their divorce rate (Fig. $6 \mathrm{H})$.

\section{Restraint hypothesis: changes in investment}

We used the residuals of the average meal size given to the chicks over the average mass gained at sea for an individual per breeding event as a proxy of its investment into reproduction. This index did not exhibit any trend with age in females but decreased linearly with age in males (Fig. 7A, GAMM: edf $=1, \mathrm{~F}=0.06, \mathrm{P}=0.809$ and edf $=1, \mathrm{~F}=5.40, \mathrm{P}=0.020$, for females and males respectively, $\mathrm{n}=984, \mathrm{~N}=238, \Delta \mathrm{AIC}=-6$ with the null model). In the absence of age-related changes in females, the age decomposition was performed only in males. Population changes mainly reflected ontogenetic changes within males (Fig. 7B).

\section{Influence of parameters to explain ontogenetic changes in breeding performances}

According to previous results, ontogenetic changes in fledging success occurred mainly at young ages with an important increase in fledging success between ages 2 to 5 and at old ages when fledging success decreased strongly between ages 16 to 20 . To explain these ontogenetic changes, we investigated whether the different parameters of performances and investment affected fledging success in these two groups of birds: young and old birds.

First, relative fledging success in young birds (ages 2 to 5) was linked to relative laying dates (LMMo": $-0.006 \pm 0.001, \mathrm{t}=-4.50, \mathrm{P}<0.001, \mathrm{n}=404, \mathrm{~N}=191, \Delta \mathrm{AIC}=-6$ with the null model and LMMQ : $-0.010 \pm 0.001, \mathrm{t}=-6.42, \mathrm{P}<0.001, \mathrm{n}=384, \mathrm{~N}=193, \Delta \mathrm{AIC}=-26$ with the null 
model; Appendix S1: Fig. S4 A) and relative foraging performances (LMMơ: $0.002 \pm 0.001, \mathrm{t}=$

506

507

508

509

510

511

512

513

514

515

516

517

518

519

520

521

522

523

524

2.95, $\mathrm{P}=0.003, \mathrm{n}=260, \mathrm{~N}=119, \Delta \mathrm{AIC}=-6$ with the null model; LMMO: $0.004 \pm 0.001, \mathrm{t}=$

4.50, $\mathrm{P}=0.003, \mathrm{n}=225, \mathrm{~N}=104, \Delta \mathrm{AIC}=-5$ with the null model; Appendix S1: Fig. S4 B). That is breeding events of earlier laying or/and with better foraging resulted in more chicks fledged. The relationship with foraging performance held true when looking at foraging performances during guard (LMMơ: $0.003 \pm 0.001, \mathrm{t}=4.52, \mathrm{P}<0.001, \mathrm{n}=219, \mathrm{~N}=106, \Delta \mathrm{AIC}=-5$ with the null model; $\mathrm{LMM}$ : $0.003 \pm 0.001, \mathrm{t}=4.36, \mathrm{P}<0.001, \mathrm{n}=211, \mathrm{~N}=103, \Delta \mathrm{AIC}=-4$ with the null model) and post-guard (LMo": $0.002 \pm 0.001, \mathrm{t}=2.16, \mathrm{P}=0.032, \mathrm{n}=193 ;$ LMO : $0.003 \pm$ $0.001, \mathrm{t}=2.57, \mathrm{P}=0.011, \mathrm{n}=179$, individual identity random effect removed due to trivial explained variance), but not in incubation (LMMo': $0.001 \pm 0.001, \mathrm{t}=1.39, \mathrm{P}=0.165, \mathrm{n}=216$,

$\mathrm{N}=103, \Delta \mathrm{AIC}=13$ with the null model; LMMO: $0.001 \pm 0.001, \mathrm{t}=1.04, \mathrm{P}=0.301, \mathrm{n}=216$, $\mathrm{N}=103, \Delta \mathrm{AIC}=14$ with the null model). Reproductive investment, the chance to switch nest or partners was not related to relative fledging success in young birds, neither in males nor females (LMMs: all $\mathrm{P}>0.05$ and all $\triangle \mathrm{AIC}>0$; Appendix S1: Fig. S4 C-D-E), although there was a tendency of higher fledging success for females that divorced less (LMMO : $-0.272 \pm 0.143, \mathrm{t}=-$ 1.90, $\mathrm{P}=0.059, \mathrm{n}=166, \mathrm{~N}=111, \Delta \mathrm{AIC}=0.5$ with the null model; Appendix S1: Fig. S4E). When focusing on old birds (i.e. $\geq 16 \mathrm{yr}$-old), the individual identity random effect on the relative number of fledged chicks was trivial and was thus removed, resulting in the use of linear models. Relative fledging success in old birds was explained by foraging performances (LMơ:

$0.005 \pm 0.002, \mathrm{t}=2.04, \mathrm{P}=0.048, \mathrm{n}=41 ; \mathrm{LM} P: 0.007 \pm 0.003, \mathrm{t}=2.39, \mathrm{P}=0.022, \mathrm{n}=39$ 

0.007, $\mathrm{n}=29$; LMQ: $0.006 \pm 0.003, \mathrm{t}=1.79, \mathrm{P}=0.085, \mathrm{n}=30$ ) but not in incubation (LMơ: guard $\left(\mathrm{LMo}^{\mathrm{*}}: 0.002 \pm 0.001, \mathrm{t}=1.66, \mathrm{P}=0.106, \mathrm{n}=40 ; \mathrm{LM}\right.$ : $0.003 \pm 0.002, \mathrm{t}=1.39, \mathrm{P}=$ did not explain the relative number of fledglings in old birds. However, the relationships with the propensity to change nest, partner and the laying date were close to significance in females, suggesting that females changing less partners or nests or laying earlier tended to produce more fledglings (Appendix S1: Fig. S5).

Second, ontogenetic changes in a parameter at young and old ages were assessed for each individual by the slopes of the linear relationships between this parameter and age in the given age groups. Using these slopes as indicators of ontogenetic changes, we explored how ontogenetic changes in fledging success could be explained by ontogenetic changes in different performance indicators or investment. In young birds, ontogenetic changes in relative fledgling success were linked to ontogenetic changes in laying date, foraging performances during guard and investment but not by ontogenetic changes in foraging performances during incubation or post-guard nor by ontogenetic changes in divorce rates and nest changes. Indeed, the increase of relative fledging success with age was related to a decrease of laying date with age (i.e. birds laying earlier with age, LM: $-0.004 \pm 0.002, \mathrm{P}=0.021, \mathrm{n}=254$; Fig. $8 \mathrm{~A}$ ), by an increase in foraging performances during guard with age ( $\mathrm{LM}: 0.002 \pm 0.000, \mathrm{P}=0.026, \mathrm{n}=118$; Fig $8 \mathrm{~B}$ ) and by an increase in investment with age (LM: $0.005 \pm 0.002, \mathrm{P}=0.005, \mathrm{n}=84$; Fig. $8 \mathrm{C})$. At old ages, the ontogenetic decrease in relative fledging success was only explained by an increase in relative laying dates (i.e. birds laying later as they aged, $\mathrm{LM}:-0.007 \pm 0.003, \mathrm{P}=0.030, \mathrm{n}=$ 
28; Fig. 8D). Note however that the number of birds available in this age category was much lower (28 birds at maximum and down to 12 birds for some parameters due to absence of information on their foraging trips).

\section{$\underline{\text { Discussion }}$}

Age-related changes in breeding performances and the relative contribution of within-versus among-individual changes

Using an extensive dataset of more than 450 birds monitored over a 19-yr period, we confirmed that little penguins displayed classical bell-shaped age-related changes in breeding performances (Dann and Cullen 1990, Nisbet and Dann 2009). Breeding success increased until age 8 then remained relatively stable before decreasing from age 16 . The number of clutches laid per year also followed a similar age-related pattern. These changes were explained by a combination of within-individual ontogenetic changes and among-individual selective mechanisms, as highlighted by two statistical analyses (i.e. the within-individual centering method (van de Pol and Wright 2009) and the decomposition method (Rebke et al. 2010)). While within-individual ontogenetic changes seemed to play a more significant role than selection mechanisms overall, their relative importance depended on age classes. Indeed, ontogenetic changes were mostly observed from ages 2 to 5 when individual performances increased at a speedy rate. These changes were again detected after age 16 when individual performances quickly declined. Selective disappearance had a small but mostly positive effect from 2 to 8 , and became quite crucial after age 16. Thus, young and older birds that displayed lower breeding performances also disappeared earlier, coinciding with the highest adult mortality. A previous study performed at the same location on age-dependent mortality has indeed shown that mortality was higher at young ages (< age 4) but also started to increase continuously after age 9 (Sidhu et al. 2007). As 
such, the older the birds the lower is the survival. Still, Sidhu and colleagues (2007) only studied data until age 17 with small sample sizes after age 15 . As survival declines are usually restricted to the most advanced age groups in seabirds (actuarial senescence), one might expect the decrease in survival to be even higher on our last age class $(\geq 16)$.

What is usually considered a unique increase of breeding performances early in life (here from age 2 to 8 ) actually resulted from the first phase of critical ontogenetic changes from ages 2 to 5 followed by selective disappearance of individuals from ages 5 to 8 . Conversely, withinindividual changes and selective mechanisms acted in opposite directions at old ages, when selective disappearance partly buffered the strong within-individual changes. Indeed, while breeding performances decreased as birds aged, only high-quality birds survived at old ages so that the apparent decrease in performance at the population scale was lowered. Decreases in breeding performance often observed at old ages can result from the senescent reduction in bird capability (Nussey et al. 2013). Senescence (or ageing) processes are defined as "progressive and irreversible changes that impair rather than improve performances" (Monaghan et al. 2008) and usually result from an accumulation of cellular damage (see Monaghan et al. (2008) for a review). While seabirds might be specially adapted to prevent age-related tissue damage caused by reactive oxygen species and prevention of formation of harmful by-products of oxidative metabolism (Holmes et al. 2001), reproductive senescence has been reported in several seabird species (e.g. Weimerskirch (1992), Tompkins and Anderson (2019)), but not in others (Berman et al. 2009, Nisbet et al. 2020)). We concluded that the importance of a decrease in reproductive performances due to senescence might be partially masked by selective disappearance when working at the population scale. This highlights that senescence needs to be studied using longitudinal data and focused on within-individual changes (Nussey et al. 2013). Also, focusing on very old birds to investigate senescence mechanisms would mean to study only high quality birds that might display different physiological capacities from birds having died earlier. 
Male, female or pair age?

599

600

601

602

603

604

605

606

607

608

609

610

611

612

613

614

615

616

617

618

619

620

621

Age-dependent patterns in breeding performances were displayed by both males and females, in contrast with (Nisbet and Dann 2009), who did not find significant age changes in females. Whether this is due to a different study period, different sample sizes or other factors is unknown. Still, we found a positive correlation between male and female ages within a pair indicating that the age effects on individual breeding success might at least partly be due to their partner's age. This correlation was related to a combination of fidelity (birds growing old together) and assortative pairing. Such assortative pairing might result from different processes that will need further studies: pairing with birds of the same cohort or similar size, similar age spatio-temporal patterns (similar arrival dates at the breeding grounds or similar habitat; (Ferrer and Penteriani 2003)), active mate choice (Cézilly et al. 1997, Schull et al. 2018), or a combination of the two (Jouventin et al. 1999). Still, we showed that laying dates differed according to age, suggesting that birds breeding timing might influence mate association and thus explain assortative pairing.

Similarly, age-related changes in laying dates, foraging performances or the tendencies to change nest or partner were very alike in males and females, suggesting analogous mechanisms in both sexes. The only real difference observed was a slight decrease in reproductive investment with age in males, while it did not vary depending on age in females (see below).

\section{Population selective mechanisms}

While selective disappearance explained part of what was observed at the population scale, selective appearance played a minor role and was virtually trivial apart from changes from age 2 to 3. Indeed, birds breeding for the first time at age 3 had a lower breeding success than birds that had already bred at age 2 that may have benefited from the previous year's experience 
622 (Limmer and Becker 2010). For instance, in other penguin species, unpaired young birds return 623 to the colony during the mating period, possibly as a learning behaviour (Barrat 1976, Saraux et

624

625

al. 2011c). Furthermore, the higher the number of times a bird returned to the colony, the more likely it was to engage in reproduction at a young age (Saraux et al. 2011c). Here, we found birds that bred at age 2 laid earlier in the season at age 3 than birds that had not bred at 2 . However, these young but experienced birds displayed lower foraging performances in females, suggesting that females that did not reproduce and spent more time at sea might have gained more foraging experience (Ramírez et al. 2015), while males did not benefit from their extra time at sea.

\section{Constraint versus restraint hypothesis?}

Within-individual changes seemed to follow the constraint hypothesis, as breeding performances are thought to increase due to experience and development at young ages and decrease due to senescence at old ages. By contrast, the restraint hypothesis predicts that investment should increase with age (Descamps et al. 2007) either all along with an individual's life or brutally at old ages due to terminal investment (Duffield et al. 2017), leading to an increase in breeding performances. Our investment index showed no relation with age in females and a slight decrease in males. Further, no sign of terminal investment or increase in reproductive success at old ages was present, although we cannot exclude that this could be due to a sharp decrease in our sample sizes for very old birds (>18 yr-old). Thus, our results tend to refute the restraint hypothesis or at least indicate that the constraint hypothesis had a much higher impact. This finding on little penguins aligns with several studies that supported constraints rather than restraints in other birds (Forslund and Pärt 1995, Martin 1995) and mammals (Broussard et al. 2008). 
648 In the constraint hypothesis, young breeders often perform poorly due to a lack of experience and capability to find sufficient resources in breeding territories or nests or food (Curio 1983).

650 Reproduction being energetically costly, most studies have focused on the acquisition of food as 651 a critically important influence on breeding performance (Lescroël et al. 2010). As individuals get older, they might learn more efficient foraging techniques and improve chick provisioning (Curio 1983, Galbraith et al. 1999). Yet, other studies showed that the breeding phenology is the main factor affected by age, which affects breeding performances (e.g. Blums et al. (1997) in which the age effect on breeding performances disappeared after controlling for phenology). As several factors may affect breeding performance, we investigated how breeding phenology, foraging performance and nest and partner choice varied with age and whether age-related changes in these factors might explain age-related differences in breeding performance. We found that from the age of 2 to 5 , birds were able to advance their laying date ( 1.5 months), increase their foraging performances by gaining more weight at sea, and decrease their probability of changing nests ( $10 \%$ points). The increase in foraging performance could be related to increased diving efficiency as seen in this and other penguin species (Zimmer et al. 2011, Le Vaillant et al. 2012, 2013) or a change in foraging areas (Pelletier et al. 2014). As part of the learning process, young birds could sample different nests to assess nest quality before settling. They might also have limited access to high-quality nests (Sherry and Holmes 1989). Birds laying earlier and exhibiting greater foraging performances had a higher breeding success. The age-related increase in bird breeding performances was explained by both an age-related increase in foraging performances and an age-related decrease in laying dates, confirming the two main hypotheses behind the constraint theory. Reproducing earlier might give them access to better partners, nesting sites or even better match prey abundance, while higher foraging 
stage separately, we found an effect of foraging performances on breeding success during chickrearing (guard and post-guard) but not during egg incubation, confirming that chick-rearing is the most critical phase of breeding (Saraux et al. 2016).

Although the constraint hypothesis is often used to explain ontogenetic developmental changes in young birds, it also applies to the decreases in breeding performance at old ages due to the senescent decrease in bird capability (Nussey et al. 2013). While reproductive senescence has been described in birds and mammals, little is known on other biological parameters (in particular life-history traits, physiology or behaviour; Gaillard and Lemaître 2020). In this study, birds older than 16 started to lay later, had lower foraging performances, and changed nest and partner often, suggesting an overall decrease in bird breeding capability at these older ages. Yet, the ontogenetic decrease in reproductive performances at old ages (i.e., reproductive senescence) was directly related to birds laying later, but no other age-related changes in any of the parameters. While these two results might seem contradictory, it actually translates into two different processes. Reproductive senescence might be explained by a general ontogenetic decrease in bird capability (in keeping the same mate and burrow, in foraging and in matching their phenology with the optimal period). However, when comparing the strength of the reproductive senescence among individuals, birds with the largest delay in their phenology displayed the highest decrease in reproductive success, while other parameters did not affect the level of reproductive senescence. However, only a few individuals (28) were monitored above age 16 in our study and the absence of foraging data for some of them might explain why we did not find statistically significant relationships between reproductive senescence and an agerelated decrease in foraging performance. The later laying for older birds might result from carry-over effects of body mass from winter to breeding, as individuals with a higher body mass in winter are more likely to breed early in the season (Salton et al. 2015). As birds older than 16 years exhibited lower foraging performance during reproduction, they may also suffer from the 
same lower performance during winter taking them longer to reach a body mass needed for the onset of the breeding season.

699

\section{Conclusions}

Reproductive performance followed a typical age-related pattern in little penguins, rapidly increasing at young ages then remaining stable before decreasing at older ages. This pattern resulted from both within- and among-individual processes, supporting the selection and constraint hypothesis. The ontogenetic increase in performances stopped rapidly at age 5, so the remaining age-related increase related to selective disappearance. This ontogenetic increase was linked to an increase in foraging performances and an advancement of breeding phenology. By contrast, the overall birds' performances decreased at old ages as they laid eggs later, had lower foraging performances, and changed nest and partners more often. Still, the strength of reproductive senescence was only related to the importance of the delay in bird phenology with age. Finally, our results did not support the restraint hypothesis in the absence of a terminal increase in reproductive performances and age-related changes in the investment index.

\section{Acknowledgements}

We wish to sincerely thank Steve Dobson for his comments on this manuscript, as well as Kelly Edwards for preliminary analyses. We thank Phillip Island Nature Parks for their continued support of penguin research. The long-term dataset received several funding sources over the years: the Penguin Foundation, Australian Academy of Science, Australian Research Council, Australian Antarctic Division, Kean Electronics and ATT Kings. The collaboration between CNRS and Phillip Island Nature Parks also received the financial support of CNRS through the International Emerging Action MECAPOP. We sincerely thank Paula Wasiak, Leanne Renwick, Marg Healy, Alona Charuvi, Marjolein van Polaten Petel, Ross Holmberg, and several past students and volunteers for their tireless support in collecting these data. The study was 
conducted with research permits issued by the Department of Environment, Land, Water and Planning, Victorian State Government, Australia, and ethics approvals from the Animal Ethics Committee of Phillip Island Nature Parks.

\section{References}

Arnould, J. P. Y., P. Dann, and J. M. Cullen. 2004. Determining the sex of Little Penguins (Eudyptula minor) in northern Bass Strait using morphometric measurements. Emu Austral Ornithology 104:261-265.

Balbontin, J., I. G. Hermosell, A. Marzal, M. Reviriego, F. De Lope, and A. P. Moller. 2007. Age-related change in breeding performance in early life is associated with an increase in competence in the migratory barn swallow Hirundo rustica. Journal of Animal Ecology 76:915-925.

Barrat, A. 1976. Quelques aspects de la biologie et de l'écologie du manchot royal (Aptenodytes patagonicus) des îles Crozet 40:107-147.

Bergeron, P., R. Baeta, F. Pelletier, D. Réale, and D. Garant. 2011. Individual quality: tautology or biological reality? Journal of Animal Ecology 80:361-364.

Berman, M., J.-M. Gaillard, and H. Weimerskirch. 2009. Contrasted patterns of age-specific reproduction in long-lived seabirds. Proceedings of the Royal Society B: Biological Sciences 276:375-382.

Blums, P., G. R. Hepp, and A. Mednis. 1997. Age-specific reproduction in three species of european ducks. Page The Auk.

Bowen, W. D., S. J. Iverson, J. I. McMillan, and D. J. Boness. 2006. Reproductive performance in grey seals: Age-related improvement and senescence in a capital breeder. Journal of Animal Ecology 75:1340-1351. 
Broussard, D. R., F. S. Dobson, and J. O. Murie. 2008. Previous experience and reproductive investment of female Columbian ground squirrels. Journal of Mammalogy 89:145-152.

Broussard, D. R., T. S. Risch, F. S. Dobson, and J. O. Murie. 2003. Senescence and age-related reproduction of female Columbian ground squirrels. Journal of Animal Ecology 72:212219.

Catchpole, C. K., and J. F. Phillips. 1992. Territory quality and reproductive success in the Dartford warbler Sylvia undata in Dorset, England. Biological Conservation 61:209-215.

Cézilly, F., V. Boy, C. J. Tourenq, and A. R. Johnson. 1997. Age-assortative pairing in the Greater Flamingo Phoenicopterus ruber roseus. Ibis 139:331-336.

Chaurand, T., and H. Weimerskirch. 1994. The Regular Alternation of Short and Long Foraging Trips in the Blue Petrel Halobaena caerulea: A Previously Undescribed Strategy of Food Provisioning in a Pelagic Seabird. The Journal of Animal Ecology 63:275.

Chiaradia, A., and K. Kerry. 1999. Daily nest attendance and breeding performance in the little penguin Eudyptula minor at Phillip Island, Australia. Marine ornithology 27:13-20.

Chiaradia, A., and I. C. T. Nisbet. 2006. Plasticity in parental provisioning and chick growth in Little Penguins Eudyptula minor in years of high and low breeding success. Ardea 94:257270.

Clutton-Brock, T. 1984. Reproductive effort and terminal investment in iteroparous animals. American Naturalist 123:212-229.

Clutton-Brock, T. 1988. Reproductive Success: Studies of Individual Variation in Contrasting breeding systems. University of Chicago Press, Chicago.

Collins, M., J. M. Cullen, and P. Dann. 1999. Seasonal and annual foraging movements of little penguins from Phillip Island, Victoria. Wildlife Research 26:705-721. 
Coltman, D. W., M. Festa-Bianchet, J. T. Jorgenson, and C. Strobeck. 2002. Age-dependent sexual selection in bighorn rams. Proceedings of the Royal Society B: Biological Sciences 269:165-172.

Cunningham, J. T., M. Le Vaillant, A. J. Gaston, Y. Ropert-Coudert, A. Kato, S. R. Jacobs, and K. H. Elliott. 2017. Reduced activity in middle-aged thick-billed murres: evidence for age related trends in fine-scale foraging behaviour. Animal Behaviour 126:271-280.

Curio, E. 1983. Why de young birds reproduce less well? Ibis 125:400-404.

Daniel, T. A., A. Chiaradia, M. Logan, G. P. Quinn, and R. D. Reina. 2007. Synchronized group association in little penguins, Eudyptula minor. Animal Behaviour 74:1241-1248.

Dann, P., M. Carron, B. Chambers, L. Chambers, T. Dornom, A. McLaughlin, B. Sharp, M. E. Talmage, R. Thoday, and S. Unthank. 2005. Longevity in Little Penguins Eudyptula minor. Marine Ornithology 33:71-72.

Dann, P., and J. M. Cullen. 1990. Survival, patterns of reproduction, and lifetime reproductive output in Little Blue Penguins. Pages 63-84 Penguin Biology. Academic Press.

Daunt, F., S. Wanless, M. P. Harris, L. Money, and P. Monaghan. 2007. Older and wiser: improvements in breeding success are linked to better foraging performance in European shags. Functional Ecology 21:561-567.

Descamps, S., S. Boutin, D. Berteaux, and J.-M. Gaillard. 2007. Female red squirrels fit Williams' hypothesis of increasing reproductive effort with increasing age. Journal of Animal Ecology 76:1192-1201.

Drent, R. H., and S. Daan. 1980. The prudent parent: energetic adjustments in avian breeding. Ardea 68:225-252.

Duffield, K. R., E. K. Bowers, S. K. Sakaluk, and B. M. Sadd. 2017. A dynamic threshold model 
792

Durant, J. M., D. Ø. Hjermann, G. Ottersen, and N. C. Stenseth. 2007. Climate and the match or mismatch between predator requirements and resource availability. Climate Research $33: 271-283$.

Fay, R., J. Martin, and F. Plard. 2020. Distinguishing within from between individual effects: How to use the within-individual centering method for quadratic pattern? BioRxive.

Ferrer, M., and I. Bisson. 2003. Age and Territory-Quality Effects on Fecundity in the Spanish Imperial Eagle (Aquila Adalberti). The Auk 120:180-186.

Ferrer, M., and V. Penteriani. 2003. A process of pair formation leading to assortative mating: Passive age-assortative mating by habitat heterogeneity. Animal Behaviour 66:137-143.

Forslund, P., and T. Pärt. 1995. Age and reproduction in birds - hypotheses and tests. Trends in Ecology \& Evolution 10:374-378.

Froy, H., R. A. Phillips, A. G. Wood, D. H. Nussey, and S. Lewis. 2013. Age-related variation in reproductive traits in the wandering albatross: evidence for terminal improvement following senescence. Ecology Letters 16:642-649.

Gaillard, J., and J. Lemaître. 2020. An integrative view of senescence in nature. Functional Ecology 34:4-16.

Galbraith, H., J. J. Hatch, I. C. T. Nisbet, and T. H. Kunz. 1999. Age-Related Changes in Efficiency among Breeding Common Terns Sterna hirundo: Measurement of Energy Expenditure Using Doubly-Labelled Water. Journal of Avian Biology 30:85.

Hatch, M. I., and D. F. Westneat. 2007. Age-related patterns of reproductive success in house sparrows Passer domesticus. Journal of Avian Biology 38:603-611. 
Holmes, D. J., and S. N. Austad. 1995. Birds as Animal Models for the Comparative Biology of Aging: A Prospectus. Journal of Gerontology: BIOLOGICAL SCIENCES 50:59-66.

Holmes, D. J., R. Flückiger, and S. N. Austad. 2001. Comparative biology of aging in birds: An update. Experimental Gerontology 36:869-883.

Jones, O. R., A. Scheuerlein, R. Salguero-Gómez, C. G. Camarda, R. Schaible, B. B. Casper, J. P. Dahlgren, J. Ehrlén, M. B. García, E. S. Menges, P. F. Quintana-Ascencio, H. Caswell, A. Baudisch, and J. W. Vaupel. 2014. Diversity of ageing across the tree of life. Nature 505:169-173.

Jouventin, P., B. Lequette, and F. S. Dobson. 1999. Age-related mate choice in the wandering albatross. Animal Behaviour 57:1099-1106.

Kato, A., Y. Ropert-Coudert, and A. Chiaradia. 2008. Regulation of trip duration by an inshore forager, the little penguin (Eudyptula minor), during incubation. The Auk 125:588-593.

Kerry, K., J. Clarke, and G. Else. 1993. The use of an automated weighing and recording system for the study of the biology of Adelie penguins (Pygoscelis Adeliae). Polar Biology 6:6275.

Kirkwood, T. B. L., and S. N. Austad. 2000. Why do we age? Nature 408:233-238.

Lescroël, A., G. Ballard, V. Toniolo, K. J. Barton, P. R. Wilson, P. O. Lyver, and D. G. Ainley. 2010. Working less to gain more: when breeding quality relates to foraging efficiency. Ecology 91:2044-2055.

Lescroël, A., K. M. Dugger, G. Ballard, and D. G. Ainley. 2009. Effects of Individual Quality, Reproductive Success and Environmental Variability on Survival of a Long-Lived Seabird. Journal of Animal Ecology 78:798-806.

Levins, R. 1968. Evolution in changing environments; some theoretical explorations. Princeton 
837

838

839

840

841

842

Limmer, B., and P. H. Becker. 2010. Improvement of reproductive performance with age and breeding experience depends on recruitment age in a long-lived seabird. Oikos 119:500507.

Marchetti, K., and T. Price. 1989. Differences in the foraging of juvenile and adult birds: the importance of developmental constraints. Biological Reviews - Cambridge Philosophical Society 64:51-70.

Martin, K. 1995. Patterns and mechanisms for age-dependent reproduction and survival in birds. Integrative and Comparative Biology 35:340-348.

Mauck, R. A., C. E. Huntington, and P. F. Doherty Jr. 2012. Experience versus effort: what explains dynamic heterogeneity with respect to age? Oikos 121:1379-1390.

Mauck, R. A., C. E. Huntington, and T. C. Grubb. 2004. Age-specific reproductive success: evidence for the selection hypothesis. Evolution 58:880-885.

McCleery, R. ., C. . Perrins, B. . Sheldon, and A. Charmantier. 2008. Age-specific reproduction in a long-lived species: the combined effects of senescence and individual quality. Proceedings of the Royal Society B: Biological Sciences 275:963-970.

Monaghan, P., A. Charmantier, D. H. Nussey, and R. E. Ricklefs. 2008. The evolutionary ecology of senescence. Functional Ecology 22:371-378.

Nisbet, I. C. T., and P. Dann. 2009. Reproductive performance of little penguins Eudyptula minor in relation to year, age, pair-bond duration, breeding date and individual quality. Journal of Avian Biology 40:296-308.

Nisbet, I. C. T., D. Iles, A. Kaneb, C. S. Mostello, and S. Jenouvrier. 2020. Breeding performance of Common Terns (Sterna hirundo) does not decline among older age classes. 
860

861

862

863

864

865

866

867

868

869

870

871

872

873

874

875

876

877

878

879

880

881

Nur, N. 1988. The consequences of brood size for breeding blue tits. III. Measuring the cost of reproduction: survival, future fecondity, and differential dispersal. Evolution 42:351-362.

Nussey, D. H., T. Coulson, M. Festa-Bianchet, and J.-M. Gaillard. 2008. Measuring Senescence in Wild Animal Populations: Towards a Longitudinal Approach. Functional Ecology 22:393-406.

Nussey, D. H., H. Froy, J. F. Lemaitre, J. M. Gaillard, and S. N. Austad. 2013. Senescence in natural populations of animals: Widespread evidence and its implications for biogerontology. Ageing Research Reviews 12:214-225.

Orgeret, F., H. Weimerskirch, and C.-A. Bost. 2016. Early diving behaviour in juvenile penguins: improvement or selection processes. Biology Letters 12:20160490.

Part, T. 1995. Does breeding experience explain increased reproductive success with age? An experiment. Proceedings of the Royal Society of London. Series B: Biological Sciences 260:113-117.

Pärt, T. 2001. The effects of territory quality on age-dependent reproductive performance in the northern wheatear, Oenanthe oenanthe. Animal Behaviour 62:379-388.

Part, T., L. Gustafsson, and J. Moreno. 1992. "Terminal investment” and a sexual conflict in the collared flycatcher (Ficedula albicollis). The American Naturalist 140:868-882.

Pelletier, L., A. Chiaradia, A. Kato, and Y. Ropert-Coudert. 2014. Fine-scale spatial age segregation in the limited foraging area of an inshore seabird species, the little penguin. Oecologia 176:399-408.

Pianka, E. R., and W. S. Parker. 1975. Age-Specific Reproductive Tactics. The American Naturalist 109:453-464. 
van de Pol, M., and S. Verhulst. 2006. Age-Dependent Traits: A New Statistical Model to Separate Within- and Between-Individual Effects. The American Naturalist 167:766-773.

van de Pol, M., and J. Wright. 2009. A simple method for distinguishing within- versus betweensubject effects using mixed models. Animal Behaviour 77:753-758.

Ramírez, F., M. G. Forero, K. A. Hobson, and A. Chiaradia. 2015. Older female little penguins Eudyptula minor adjust nutrient allocations to both eggs. Journal of Experimental Marine Biology and Ecology 468:91-96.

Rebke, M., T. Coulson, P. H. Becker, and J. W. Vaupel. 2010. Reproductive improvement and senescence in a long-lived bird. Proceedings of the National Academy of Sciences of the United States of America 107:7841-7846.

Reed, T. E., S. Jenouvrier, and M. E. Visser. 2013. Phenological mismatch strongly affects individual fitness but not population demography in a woodland passerine. Journal of Animal Ecology 82:131-144.

Reed, T. E., L. E. B. Kruuk, S. Wanless, M. Frederiksen, E. J. A. Cunningham, and M. P. Harris. 2008. Reproductive Senescence in a Long-Lived Seabird: Rates of Decline in Late-Life Performance Are Associated with Varying Costs of Early Reproduction. The American Naturalist 171:E89--E101.

Reid, J. M., E. M. Bignal, S. Bignal, D. I. McCracken, M. I. Bogdanova, and P. Monaghan. 2008. Investigating Patterns and Processes of Demographic Variation: Environmental Correlates of Pre-Breeding Survival in Red-Billed Choughs Pyrrhocorax pyrrhocorax. Journal of Animal and Veterinary Advances 77:777-788.

Reilly, P. N., and J. M. Cullen. 1979. The little penguin eudyptula minor in Victoria, I: Mortality of adults. Emu 79:97-102. 
Reilly, P. N., and J. M. Cullen. 1981. The little penguin eudyptula minor in Victoria, II: Breeding. Emu 81:1-19.

Robinson, S., A. Chiaradia, and M. A. Hindell. 2005. The effect of body condition on the timing and success of breeding in Little Penguins Eudyptula minor. Ibis 147:483-489.

Ropert-Coudert, Y., R. P. Wilson, F. Daunt, and A. Kato. 2004. Patterns of energy acquisition by a central place forager: benefits of alternating short and long foraging trips. Behavioral Ecology 15:824-830.

Rutz, C., M. J. Whittingham, and I. Newton. 2006. Age-dependent diet choice in an avian top predator. Proceedings of the Royal Society B: Biological Sciences 273:579-586.

Salton, M., C. Saraux, P. Dann, and A. Chiaradia. 2015. Carry-over body mass effect from winter to breeding in a resident seabird, the little penguin. Royal Society Open Science 2:140390.

Saraux, C., A. Chiaradia, Y. Le Maho, and Y. Ropert-Coudert. 2011a. Everybody needs somebody: unequal parental effort in little penguins. Behavioral Ecology 22:837-845.

Saraux, C., A. Chiaradia, M. Salton, P. Dann, and V. A. Viblanc. 2016. Negative effects of wind speed on individual foraging performance and breeding success in little penguins. Ecological Monographs 86:61-77.

Saraux, C., S. M. Robinson-Laverick, Y. Le Maho, Y. Ropert-Coudert, and A. Chiaradia. 2011 b. Plasticity in foraging strategies of inshore birds: how Little Penguins maintain body reserves while feeding offspring. Ecology 92:1909-1916.

Saraux, C., V. A. Viblanc, N. Hanuise, Y. Le Maho, and C. Le Bohec. 2011c. Effects of individual pre-fledging traits and environmental conditions on return patterns in juvenile king penguins. PloS one 6:e20407. 
Schull, Q., V. A. Viblanc, F. S. Dobson, J. P. Robin, S. Zahn, R. Cristofari, P. Bize, and F. Criscuolo. 2018. Assortative pairing by telomere length in King Penguins (Aptenodytes patagonicus) and relationships with breeding success. Canadian Journal of Zoology 96:639-647.

Schwartz, A. M., T. A. Baird, and D. K. Timanus. 2007. Influence of Age and Prior Experience on Territorial Behavior and the Costs of Defense in Male Collared Lizards. Ethology 113:9-17.

Sherry, T. W., and R. T. Holmes. 1989. Age-specific social dominance affects habitat use by breeding American redstarts (Setophaga ruticilla): a removal experiment. Behavioral Ecology and Sociobiology 25:327-333.

Sidhu, L. A., E. A. Catchpole, and P. Dann. 2007. Mark-recapture-recovery modeling and agerelated survival in little penguins (Eudyptula minor). The Auk 124:815-827.

Siikamäki. 1995. Habitat quality and reproductive traits in the Pied Flycatcher: an experiment. Ecology 76:308-312.

Spong, G. F., S. J. Hodge, A. J. Young, and T. Clutton-Brock. 2008. Factors affecting the reproductive success of dominant male meerkats. Molecular Ecology 17:2287-2299.

Stearns, S. C. 1989. Trade-Offs in Life-History Evolution. Functional Ecology 3:259.

Sutherland, D. R., and P. Dann. 2014. Population trends in a substantial colony of Little Penguins: three independent measures over three decades. Biodiversity Conservation 23:241-250.

Tompkins, E. M., and D. J. Anderson. 2019. Sex-specific patterns of senescence in Nazca boobies linked to mating system. Journal of Animal Ecology 88:986-1000.

Trivers, R. 1972. Parental investment and sexual selection. Pages 136-179 Sexual Selection and 
952

Tuljapurkar, S., U. K. Steiner, and S. H. Orzack. 2009. Dynamic heterogeneity in life histories. Ecology Letters 12:93-106.

Le Vaillant, M., C. Le Bohec, O. Prud'Homme, B. Wienecke, Y. Le Maho, A. Kato, and Y. Ropert-Coudert. 2013. How age and sex drive the foraging behaviour in the king penguin. Marine Biology 160:1147-1156.

Le Vaillant, M., R. P. Wilson, A. Kato, C. Saraux, N. Hanuise, O. Prud'Homme, Y. Le Maho, C. Le Bohec, and Y. Ropert-Coudert. 2012. King penguins adjust their diving behaviour with age. Journal of Experimental Biology 215:3685-3692.

Vaupel, J. W., and A. I. Yashin. 1985. Heterogeneity's ruses: Some surprising effects of selection on population dynamics. American Statistician 39:176-185.

Velando, A., H. Drummond, and R. Torres. 2006. Senescent birds redouble reproductive effort when ill: confirmation of the terminal investment hypothesis. Proceedings of the Royal Society B: Biological Sciences 273:1443-1448.

Viblanc, V. A., C. Saraux, J. O. Murie, and F. S. Dobson. 2016. Kin effects on energy allocation in group-living ground squirrels. Journal of Animal Ecology 85:1361-1369.

Weimerskirch, H. 1992. Reproductive effort in long-lived birds: age-specific patterns of condition, reproduction and survival in the wandering albatross. Oikos 64:464.

Williams, G. C. 1966. Natural Selection, the Costs of Reproduction, and a Refinement of Lack's Principle. The American Naturalist 100:687-690.

Wilson, A. J., and D. H. Nussey. 2010. What is individual quality? An evolutionary perspective. Trends in Ecology \& Evolution 25:207-214. 

6:e16098. 
Table 1. Within and among-individual age effects on the relative number of fledglings in little 980 penguins. Main statistics associated with the 2 linear mixed models, one for males and one for 981 females.

\begin{tabular}{|c|c|c|c|c|c|c|}
\hline & Variable & Estimate $\pm \mathrm{SE}$ & t values & $P$ values & $\begin{array}{c}\text { Sum } \\
\text { Squares }\end{array}$ & Sample size \\
\hline \multirow{5}{*}{ Males } & Intercept & $-0.223 \pm 0.083$ & -2.68 & 0.008 & - & \multirow{5}{*}{$\begin{array}{c}\mathrm{n}=788, \mathrm{~N}= \\
142\end{array}$} \\
\hline & Age_center & $0.119 \pm 0.036$ & 3.27 & 0.001 & 6.69 & \\
\hline & Age_mean & $0.030 \pm 0.010$ & 2.94 & 0.004 & 5.40 & \\
\hline & Age $^{2} \_$center & $-0.007 \pm 0.002$ & -3.90 & $<0.001$ & 9.54 & \\
\hline & $\mathrm{Age}^{2} \_$mean & \multicolumn{4}{|c|}{ not selected in the best model } & \\
\hline \multirow{5}{*}{ Females } & Intercept & $-0.767 \pm 0.211$ & -3.63 & $<0.001$ & - & \multirow{5}{*}{$\begin{array}{c}\mathrm{n}=743, \mathrm{~N}= \\
141\end{array}$} \\
\hline & Age_center & $0.149 \pm 0.038$ & 3.88 & $<0.001$ & 9.49 & \\
\hline & Age_mean & $0.205 \pm 0.057$ & 3.61 & $<0.001$ & 8.23 & \\
\hline & Age $^{2} \_$center & $-0.008 \pm 0.002$ & -4.09 & $<0.001$ & 10.57 & \\
\hline & $\mathrm{Age}^{2} \_$mean & $-0.011 \pm 0.003$ & -3.32 & 0.001 & 6.94 & \\
\hline
\end{tabular}


Fig 1. Reproductive investment index calculation, as the residuals of the regression of average 987 chick meal size on average body mass change during a trip $(y=(0.95 \pm 0.03) x+(6.31 \pm 7.64)$, $\mathrm{P}<0.001, \mathrm{n}=984)$. Each point represents the average over a post-guard event. Points are transparent so that darker areas represent higher densities of points.

Fig 2. Relationships between female and male age within a pair over A) all breeding attempts, B) breeding attempts where new partners had to be found (i.e. first breeding attempts or when an individual changed partners). The average prediction from the linear mixed model is indicated with a black line on each panel.

Fig 3. Relative reproductive success according to age (mean $\pm \mathrm{SE}$ ): A) relative number of fledglings per breeding event and B) relative number of clutches per year. Males are indicated in blue and females in red. Sample sizes are indicated at the bottom of the panels for each age category. Further, the prediction and confidence interval of the GAMMs are drawn as blue and red lines and ribbons for male and female, respectively.

Fig 4. Age effects on relative number of fledglings according to the within-subject method in A) 1000 females and B) males.

Fig 5. Relative reproduction according to age. Panels A (males) and C (females) represent the observed population trend (in dark blue), as well as the age patterns we would have observed had only one process occurred in the population: light blue for ontogenetic development, red selective disappearance, and orange selective appearance. Note that age 21 was not represented for clarity purposes as only one male and one female of age 21 were present. Panels B (males) 
and $\mathrm{D}$ (females) summarise changes in relative reproduction from one age to the next by age categories according to the different processes.

1008

1009

1010

1011

1012

1013

1014

1015

1016

1017

1018

1019

1020

1021

1022

1023

1024

1025

1026

1027

1028

1029

Fig 6. Test of the constraint hypothesis. Age-related changes (mean \pm SE) in A) foraging performances, B) laying date, C) proportion of birds that changed nest burrows and D) proportion of birds that changed partners. Sample sizes are indicated per age class at the top and bottom of the panels. Blue and red lines and ribbons indicate male and female prediction along with their confidence intervals from the GAMM, while a black line and grey ribbon indicate the predictions for all individuals pooled in the absence of sex effect. The bottom panels (E, F, G and $\mathrm{H}$ ) represent the changes from one age to the next at the population level and according to ontogenetic changes, selective disappearance or selective appearance for each of the four parameters for the four different age classes highlighted in fledging success analyses. These are separated by sex for foraging performances $(E)$, laying date $(F)$ and divorce rates $(H)$, while the decomposition was run on both sexes pooled for proportion of birds changing nests $(\mathrm{G})$ in the absence of significant differences in the age-related changes of males and females. Note that selective appearance cannot be tested for the proportion of birds that changed nest or partners as these can be defined only compared to the previous year (so that birds cannot appear).

Fig. 7. Test of the restraint hypothesis. A) Age-related changes in investment into reproduction for males in blue and females in red. Mean \pm SE. Sample sizes are indicated per age class and sex at the top and bottom of the plot for males and females respectively. The GAMM prediction and confidence interval are drawn as blue line and ribbon for male, while no prediction is displayed for female in the absence of significant age-related changes in reproductive investment. B) Changes in males only from one age to the next at the population level, according to ontegenetic changes, selective disappearance or selective appearance for the four different age classes highlighted in fledging success analyses. 
1030 Fig. 8 Relationships between ontogenetic changes in relative fledging success and A) relative 1031 laying dates, B) foraging performances during guard, C) reproductive investment in young birds 1032 (ages 2 to 5) and D) relative laying dates in old birds ( $\geq 16$ yr-old). 


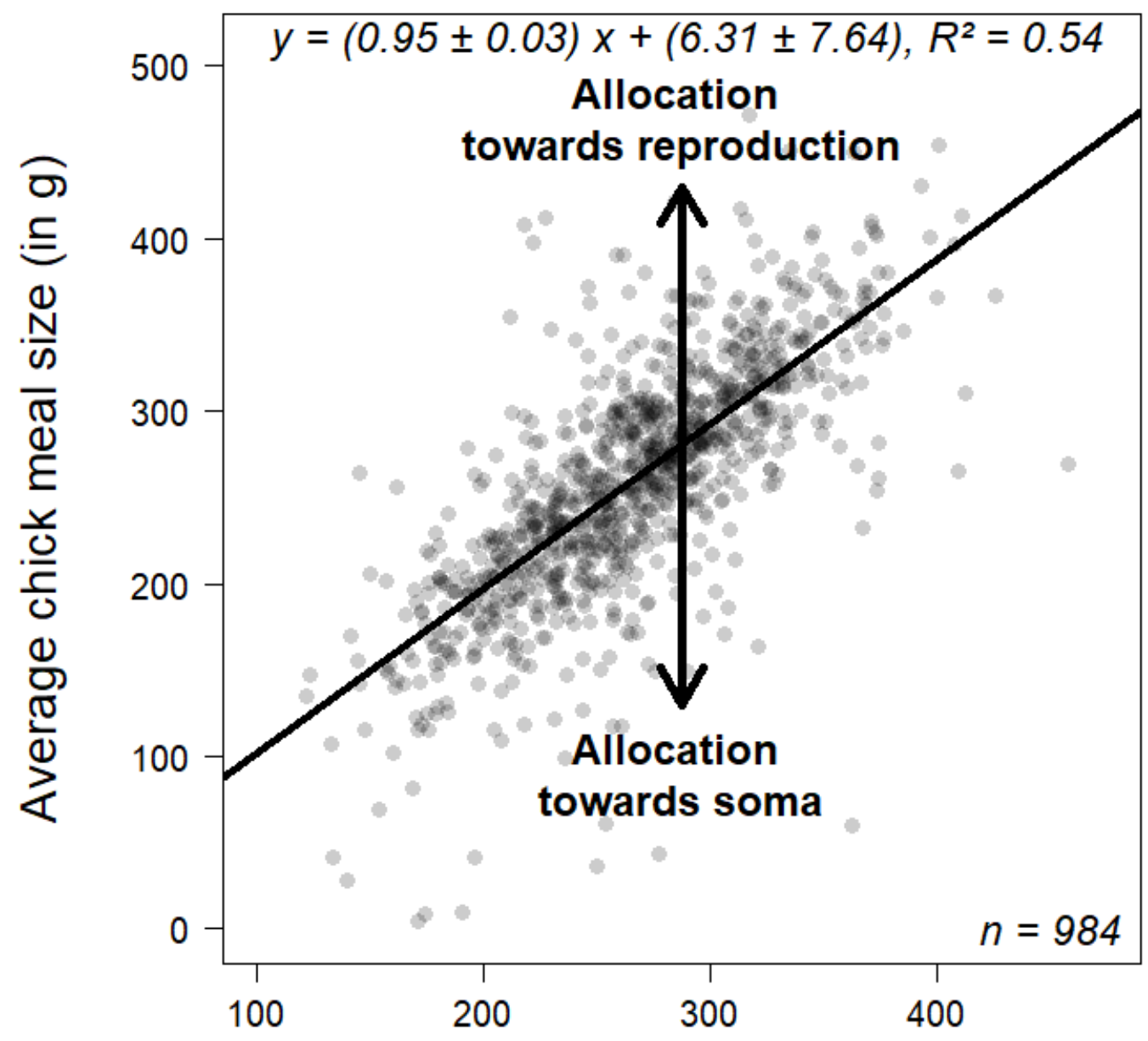

Average body mass gain per foraging trip (in g)

$1034 \quad$ Figure 1.

1035

1036 
All breeding attempts

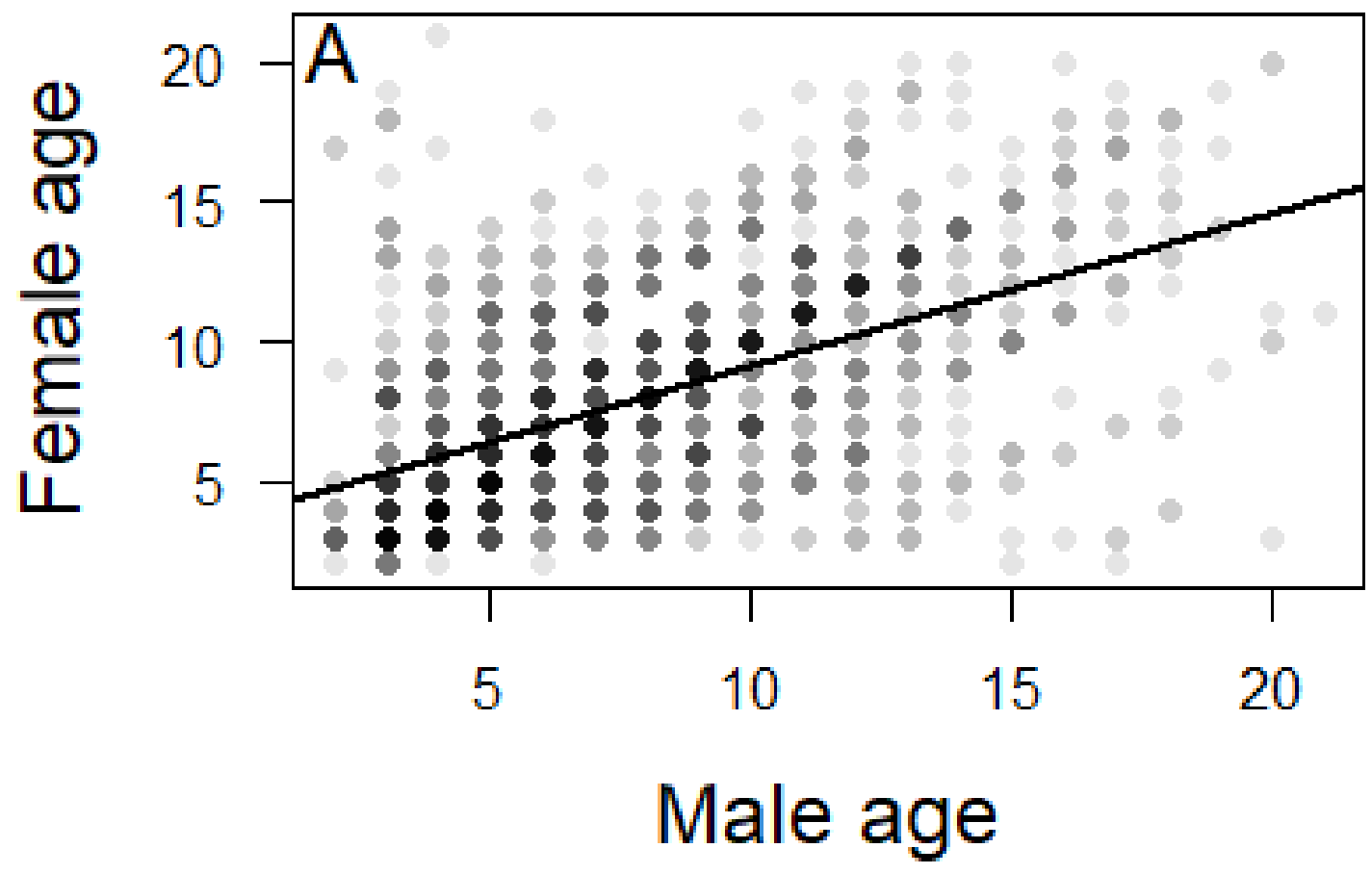

Breeding attempts with new partners

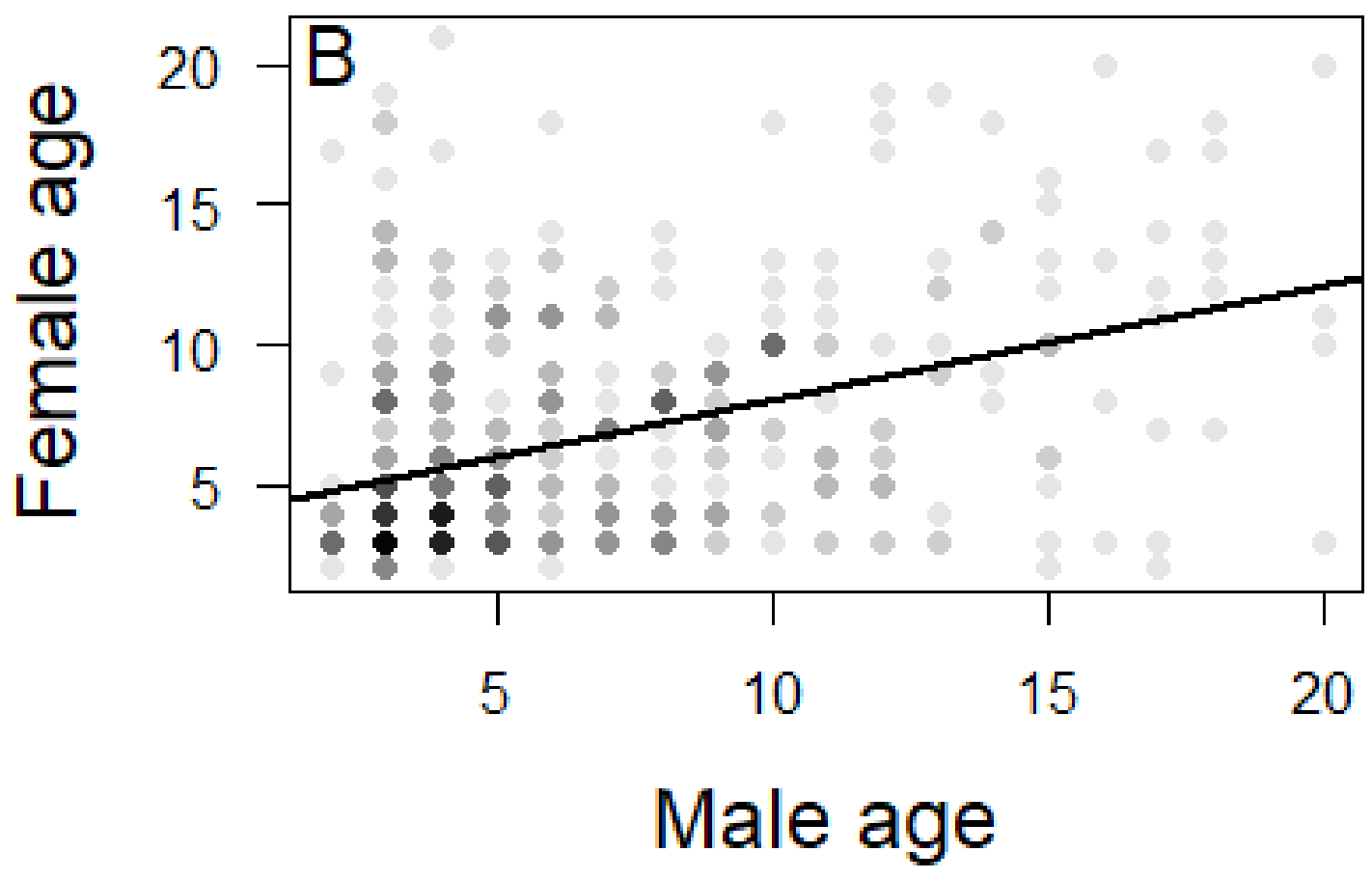

1038 Figure 2 

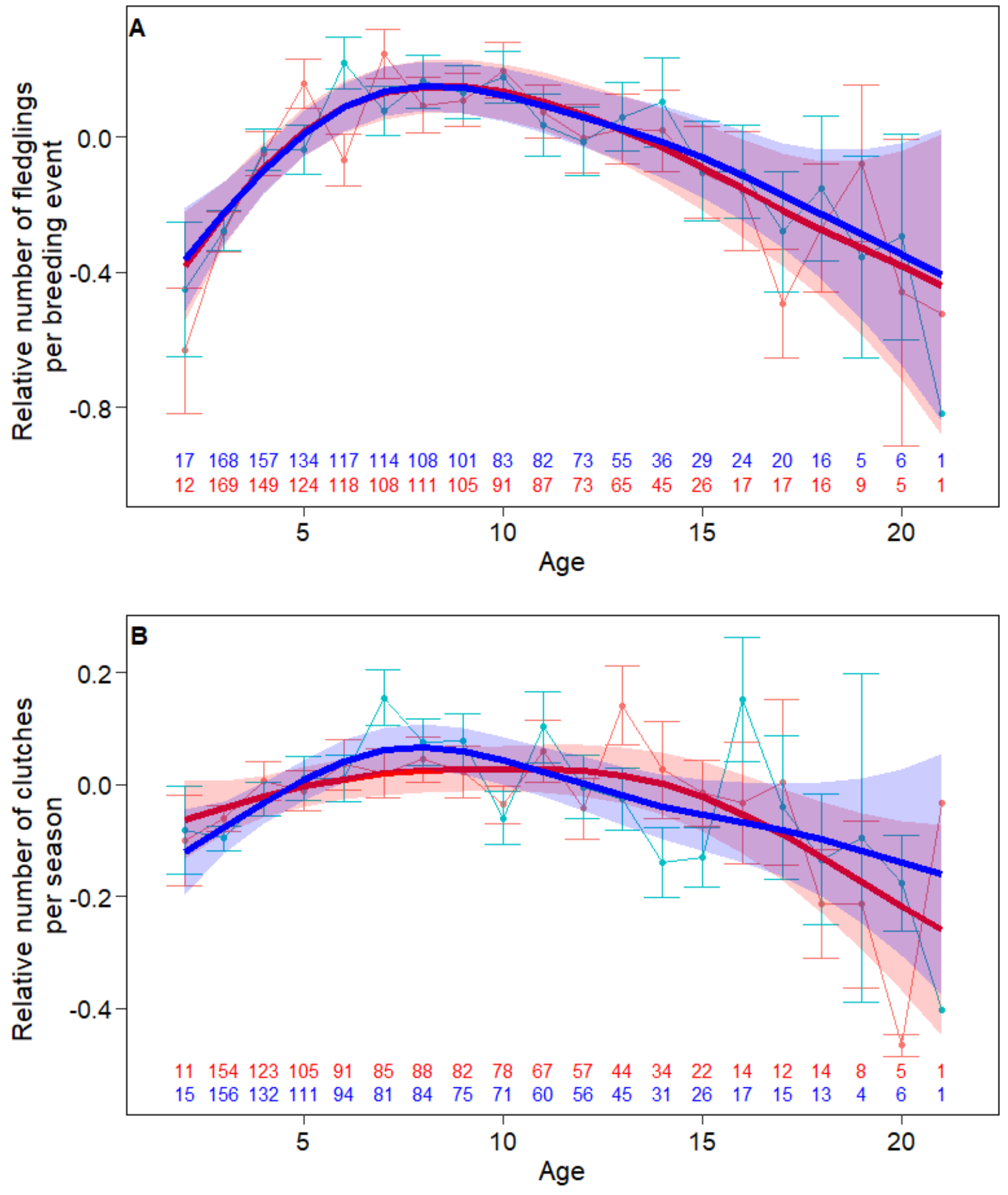

$1040 \quad$ Figure 3.

1041

1042 

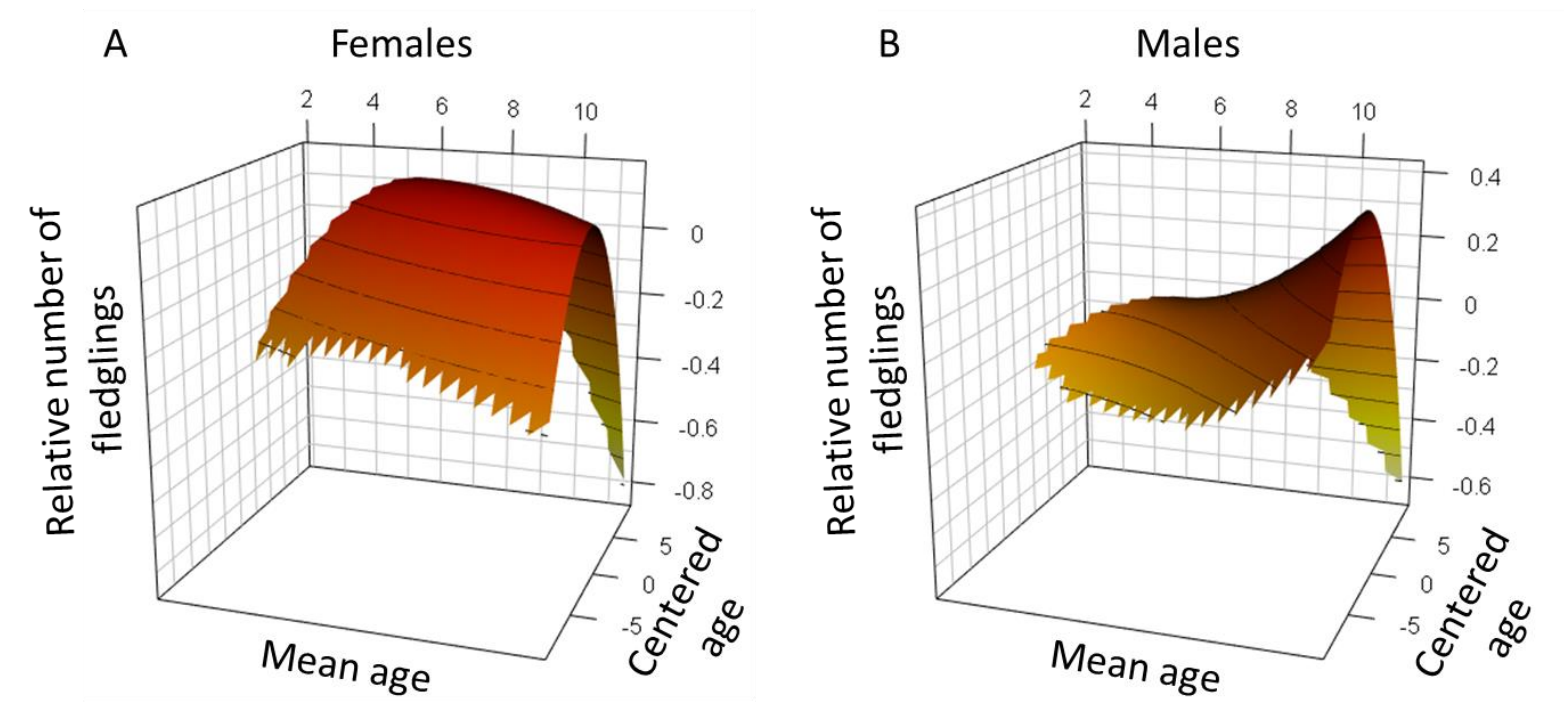

1043

1044

$1045 \quad$ Fig 4.

1046 

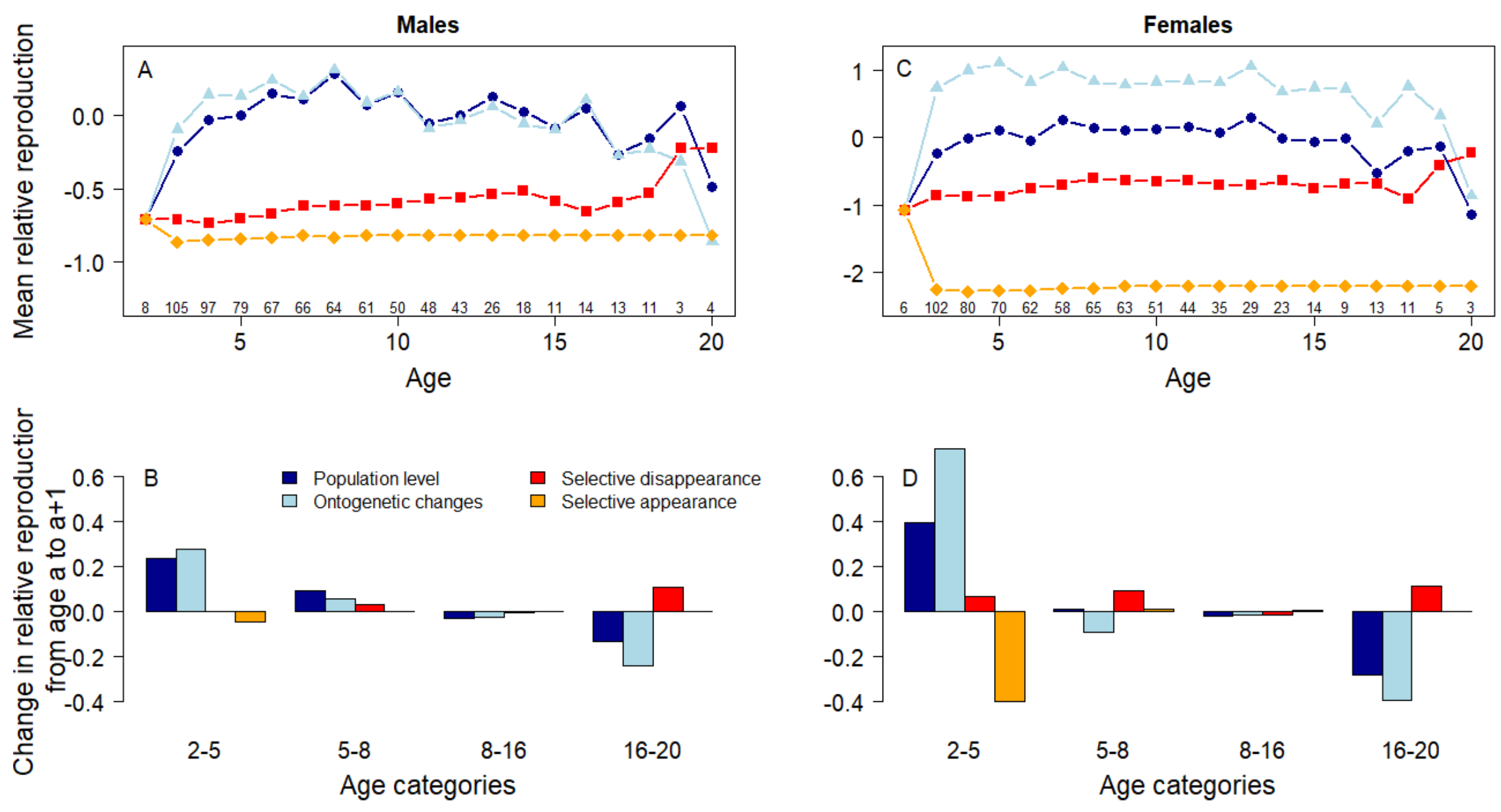

Figure 5. 
Constraint hypothesis
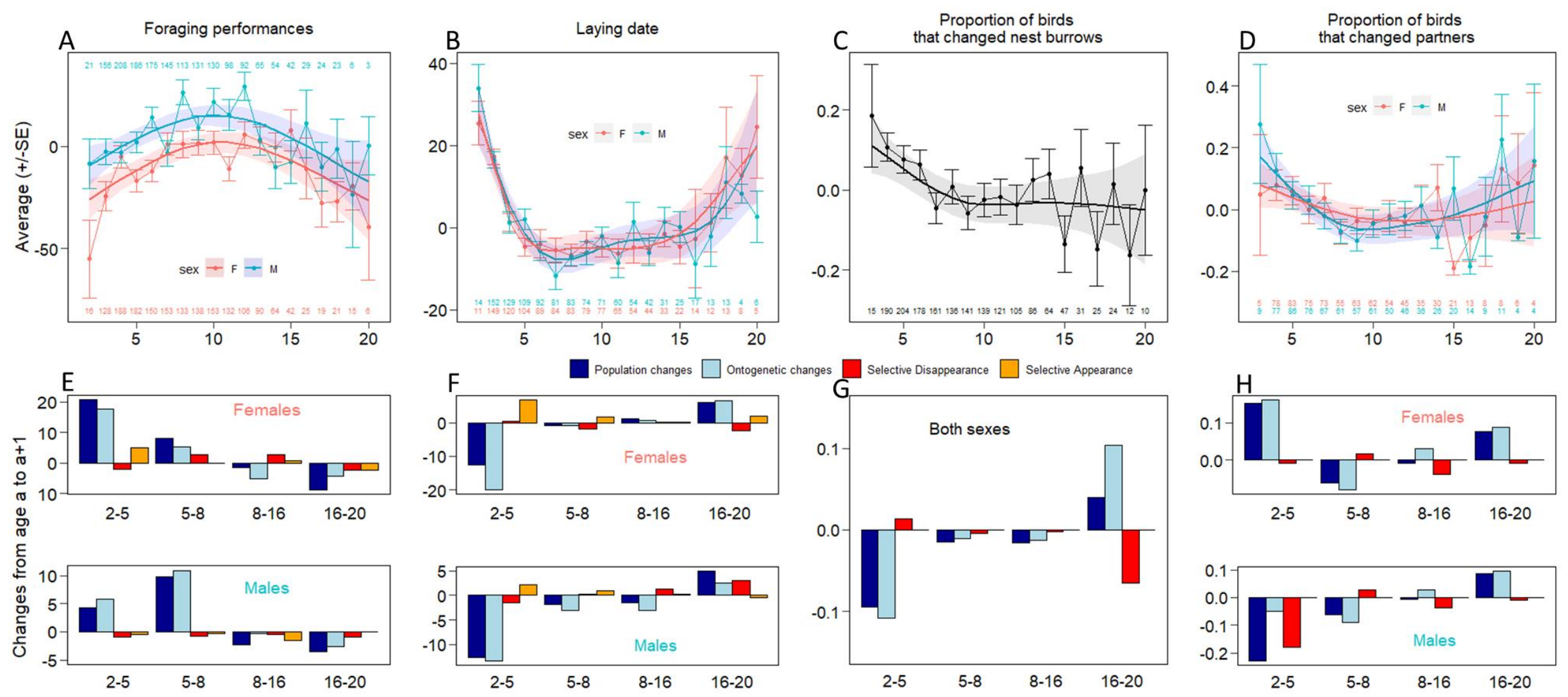

Figure 6. 

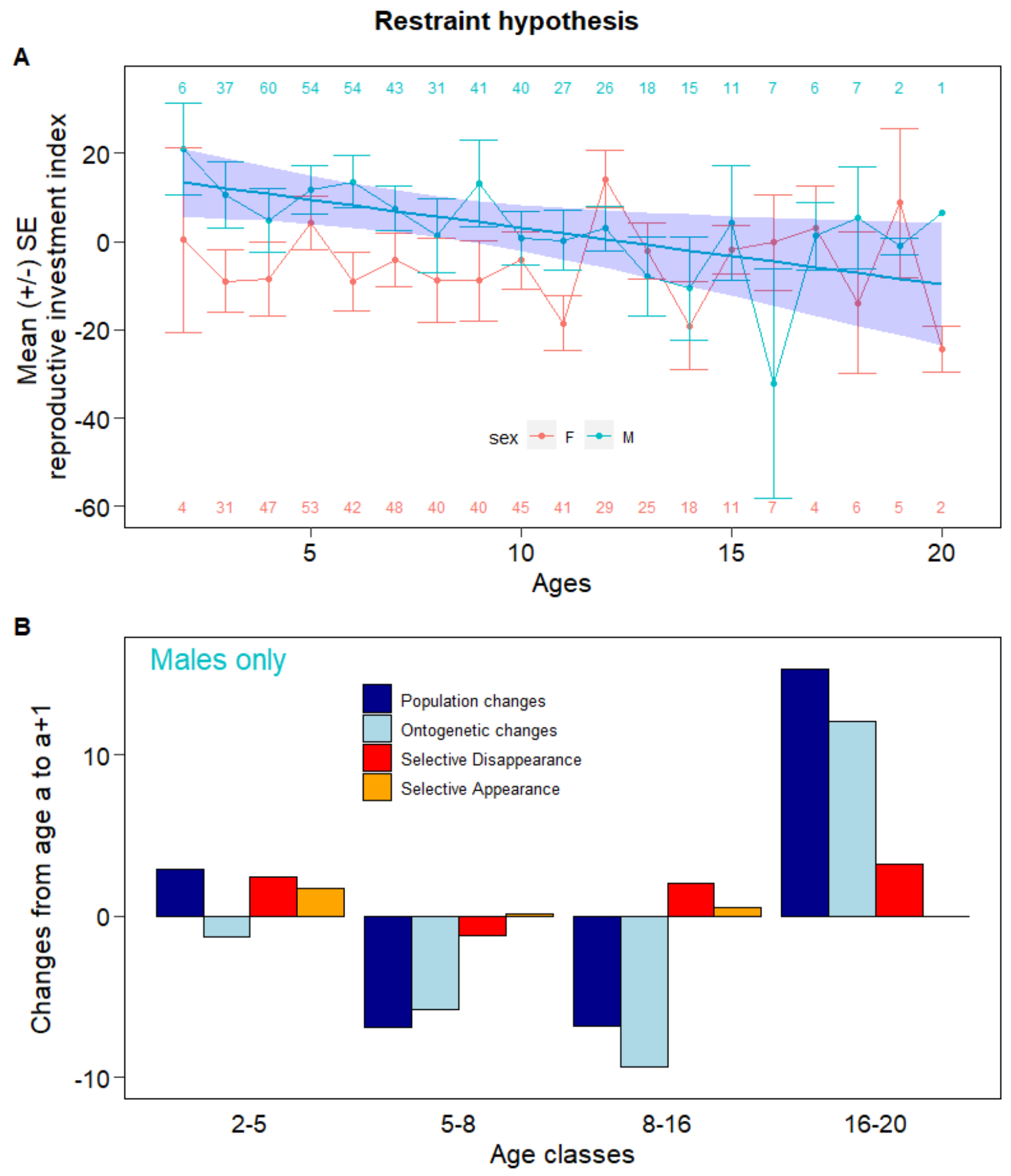

$1052 \quad$ Figure 7. 


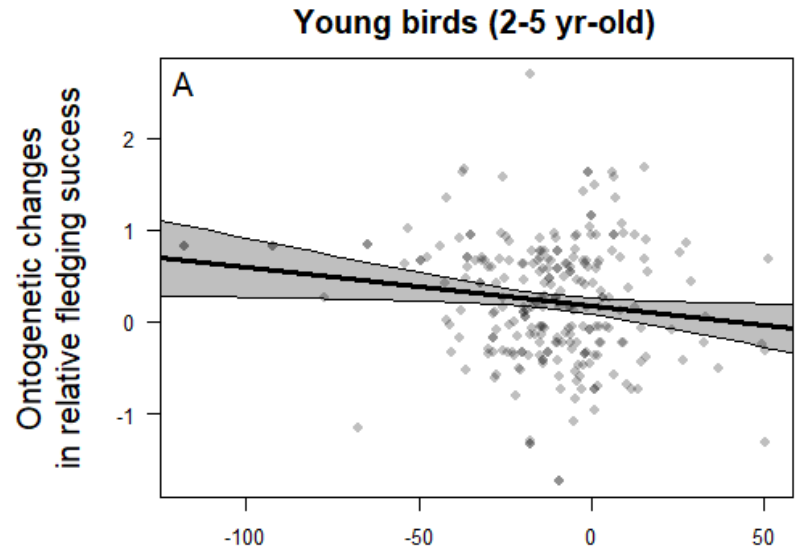

Ontogenetic changes in relative laying dates

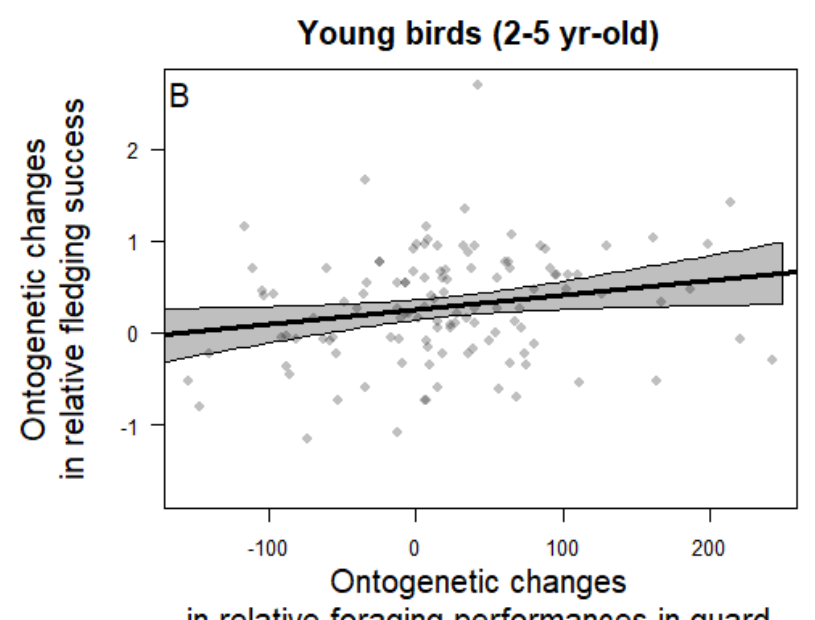
in relative foraging performances in guard

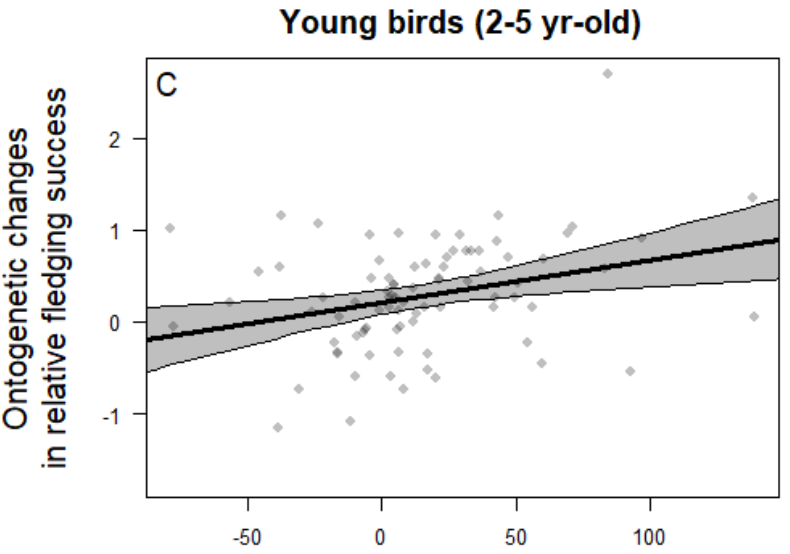

Ontogenetic changes in reproductive investment

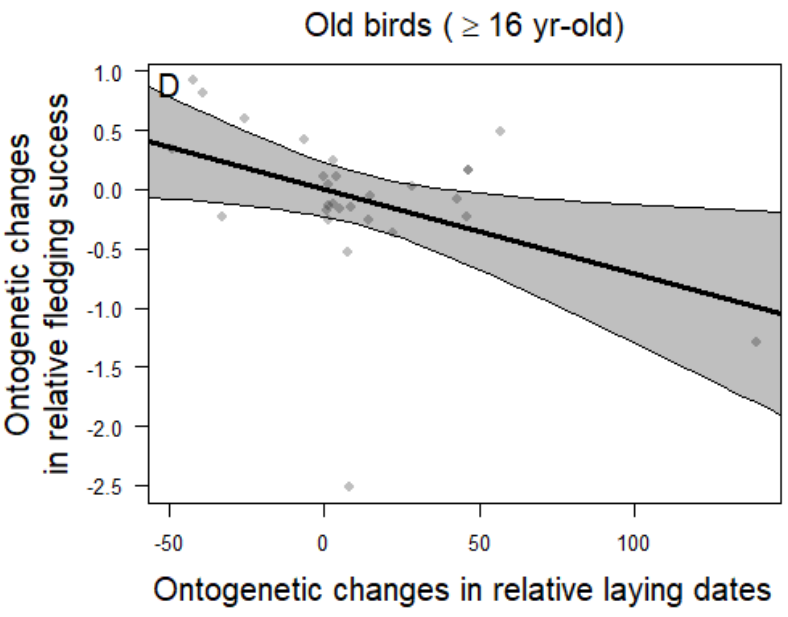

Figure 8.

1057 
1060 Saraux C., Chiaradia A. Age-related breeding success in little penguins: a result of selection and 1061 ontogenetic changes in foraging and phenology. Ecological Monographs

1062

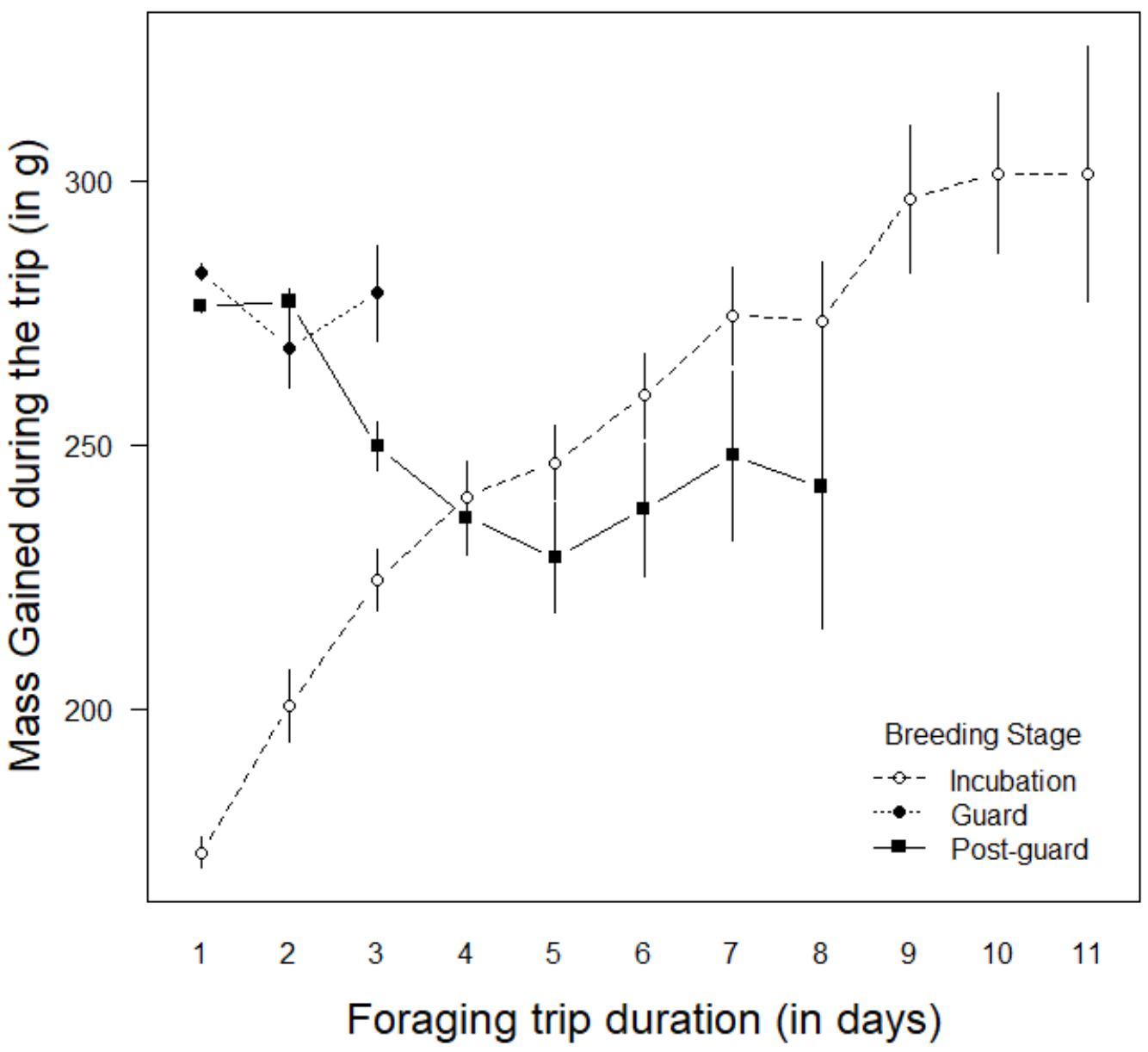

1064 Figure S1: Mean \pm SE mass gained during a foraging trip (in g) according to foraging trip 1065 duration (in days) for each breeding stage (incubation indicated with open circles and a dashed 1066 line, guard with black circles and a dotted line, and post-guard with black squares and a plain 1067 line). 

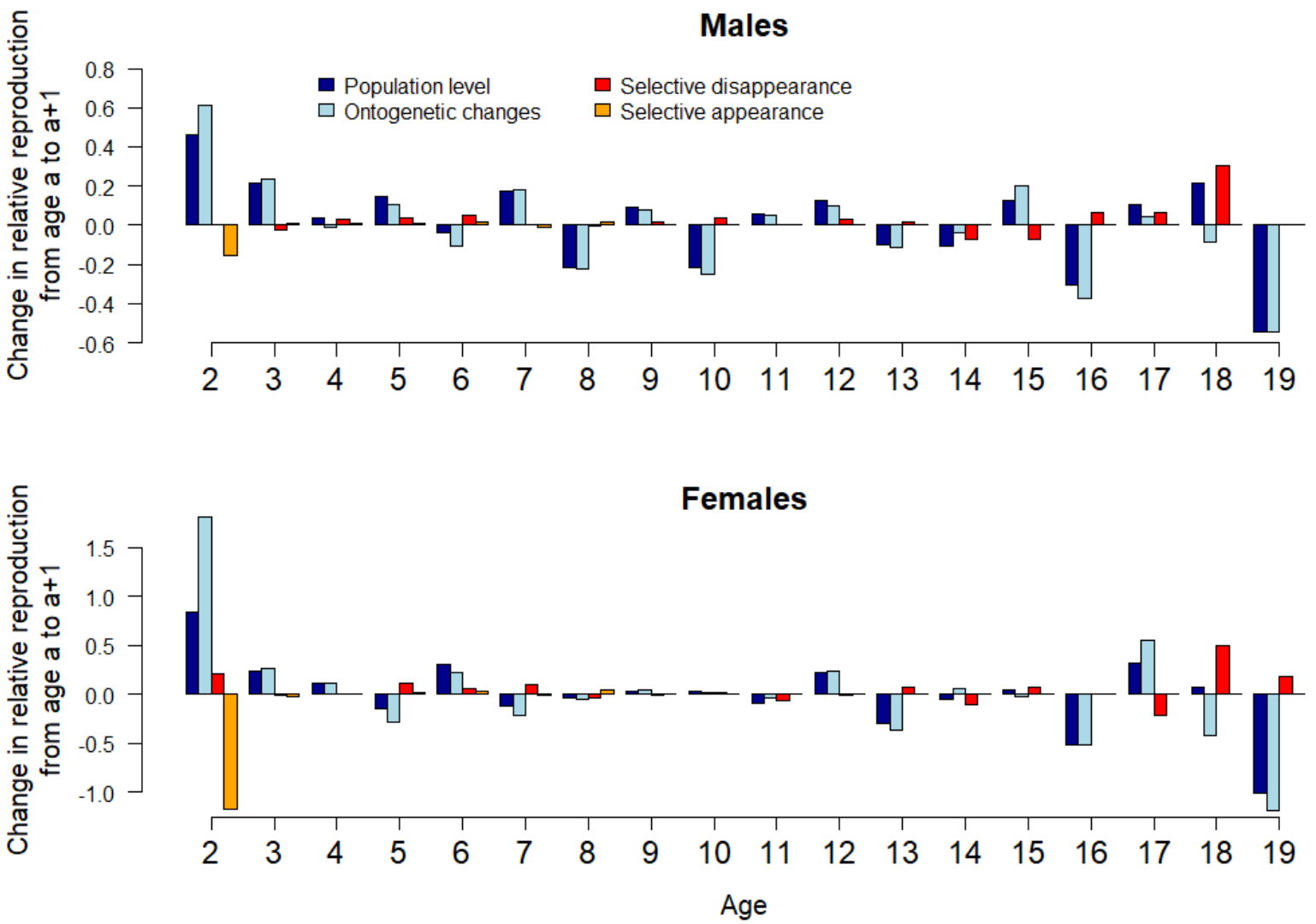

1069

1070 Figure S2. Change in relative reproduction from one age to the next at the population level (dark blue) and its decomposition in within-individual ontogenetic changes (light blue), selective 1072 disappearance (red) and selective appearance (orange) in male and female little penguins. 

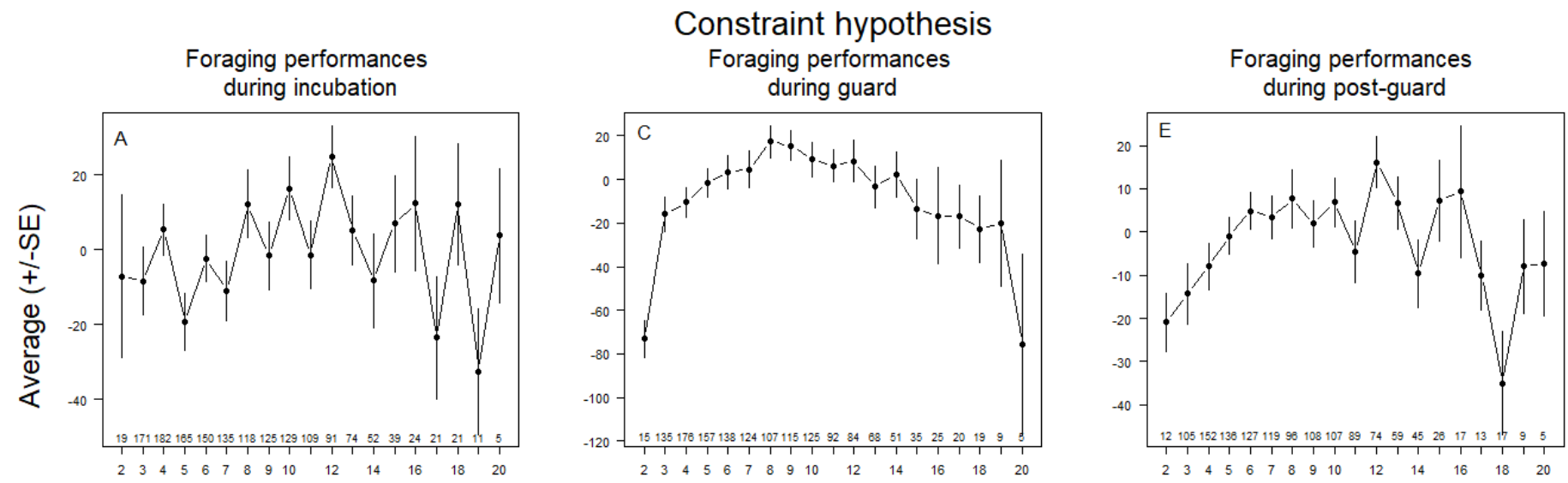

Ages

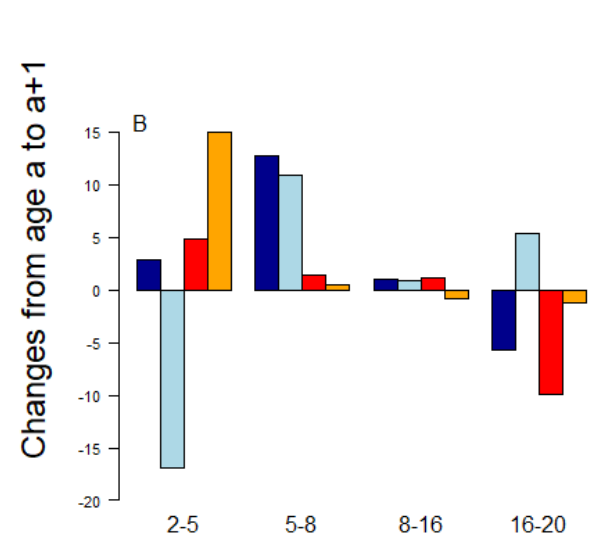

- Population level

Ontogenetic changes

\section{- Selective disappearance}
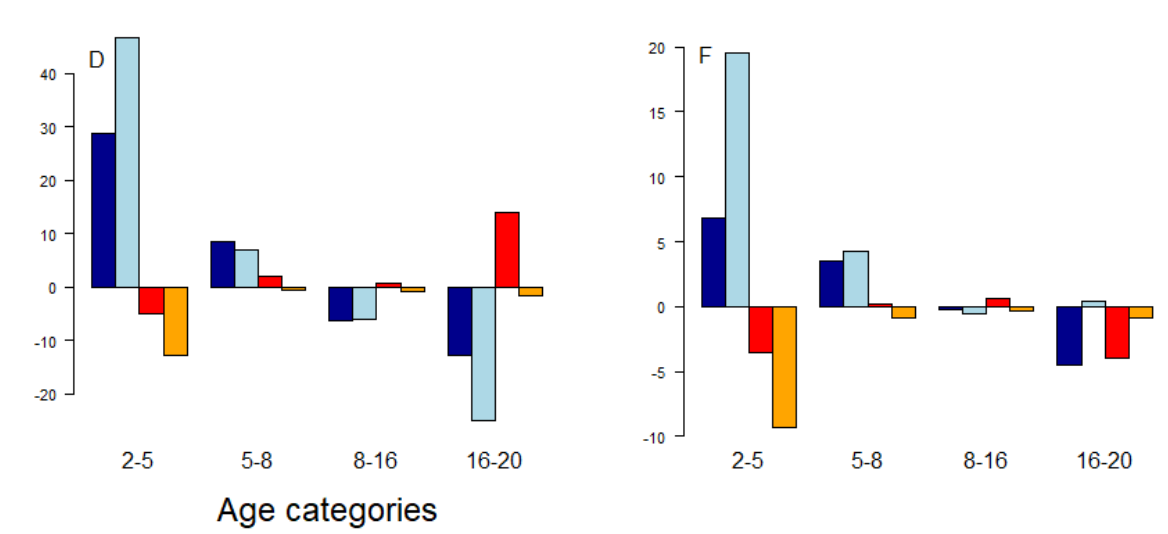

1073

1074 Figure S3. Constraint hypothesis - Foraging performances according to age during incubation (A), guard (C), and post-guard (E) and changes in 
Young birds $(2-5 \mathrm{yr}-$ old $)$
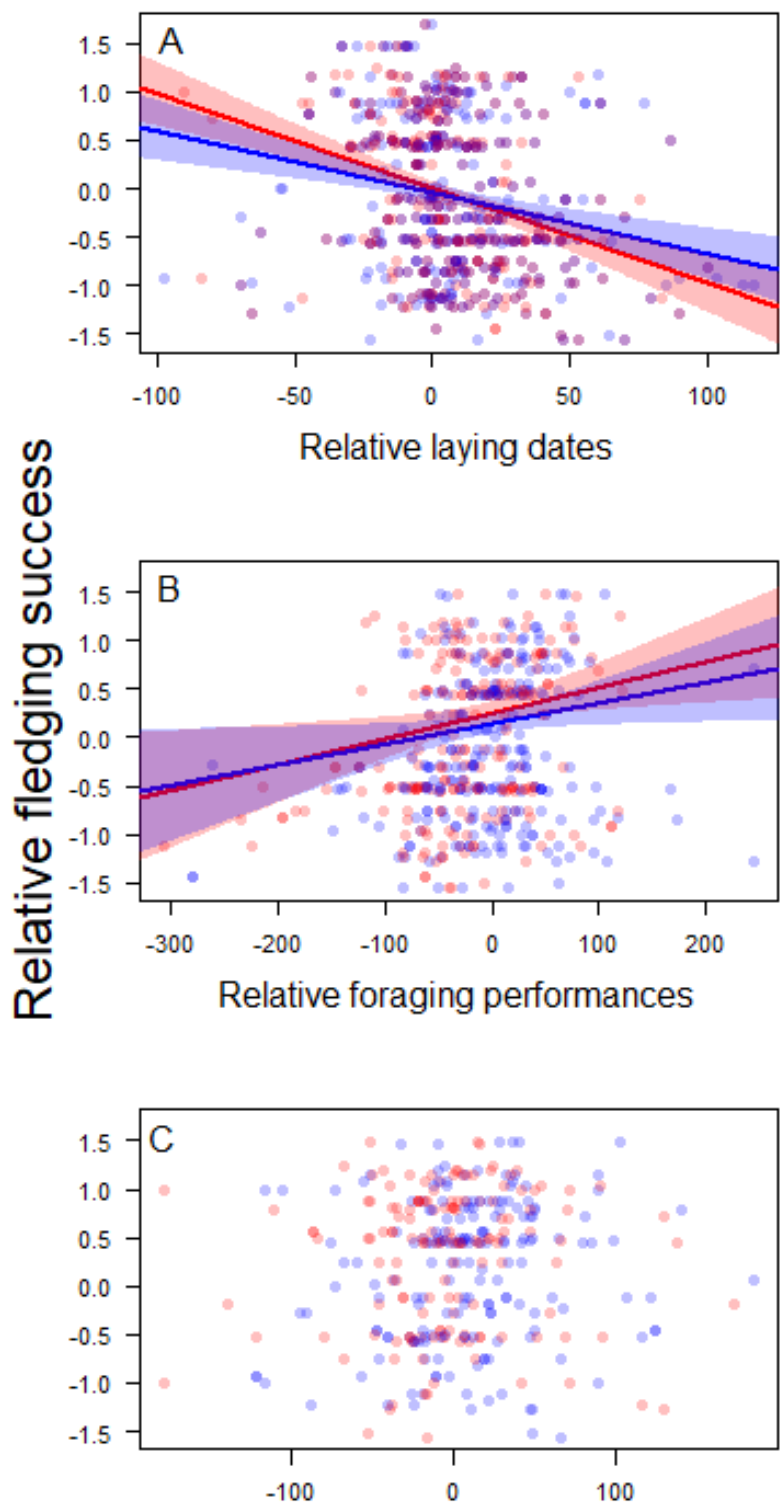

Relative reproductive investment

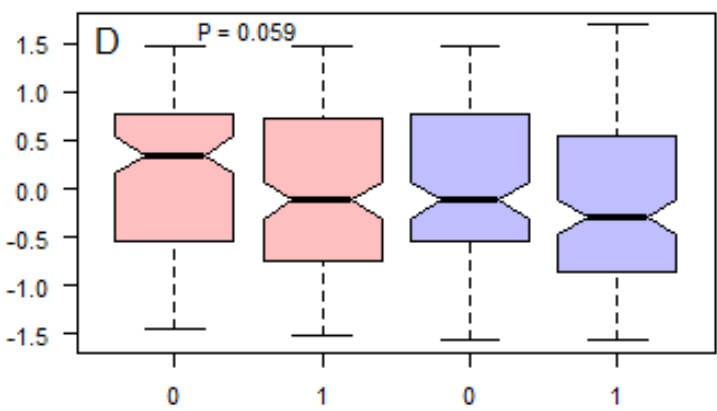

Probability of changing nest

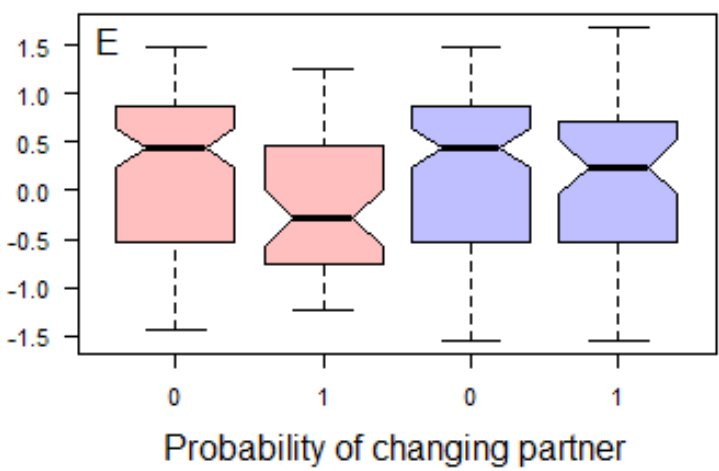

Females

Males

Figure S4. Relative fledging success according to relative laying dates (A), relative foraging 1080 performances (B) relative reproductive investment (C), divorce rate (D) and change of partner 1081 (E) in young birds (ages 2-5). Significant relationships are indicated with lines and confidence intervals around. As one P-value was close to significant, it is given in panel D. Males are shown in blue, while females are shown in red. 
Old birds (>=16 yr-old)
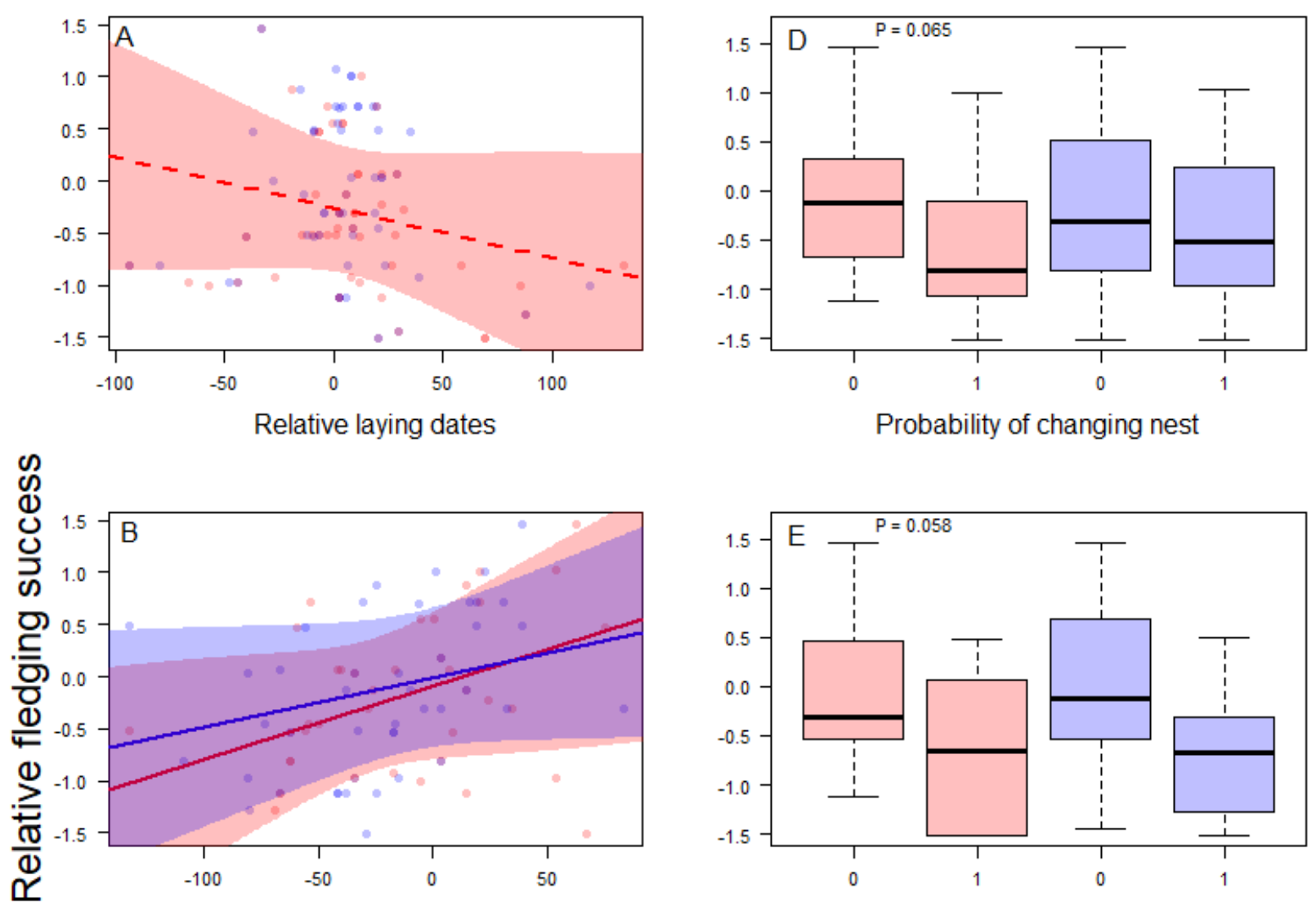

Relative foraging performances

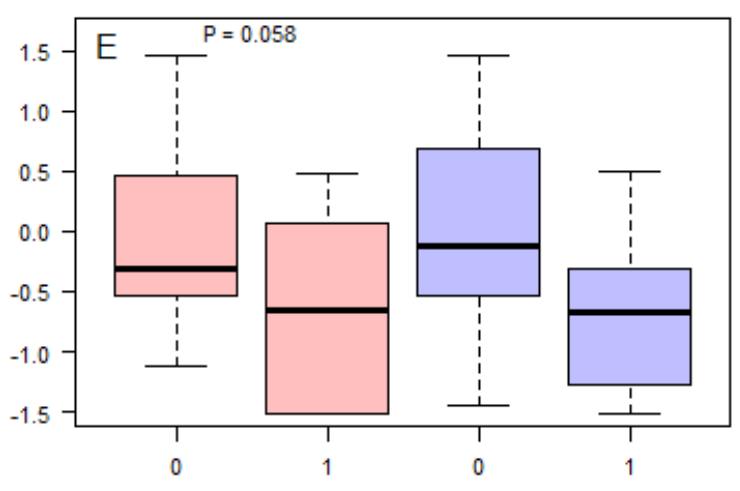

Probability of changing partner
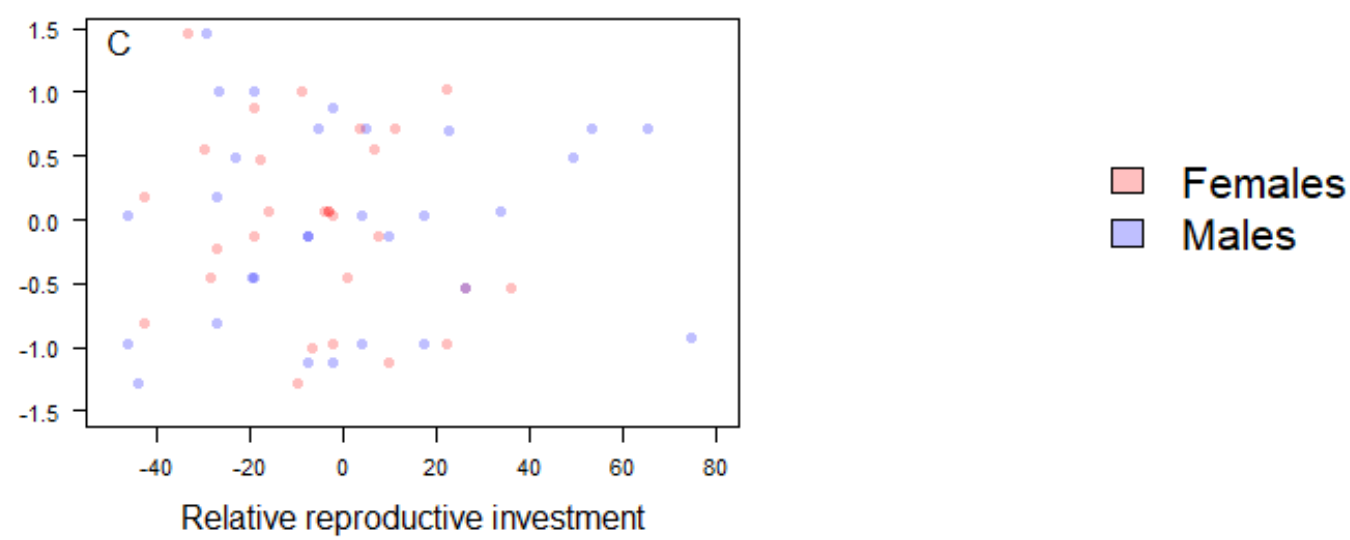

Figure S5. Relative fledging success according to relative laying dates (A), relative foraging performances (B) relative reproductive investment (C), divorce rate (D) and change of partner (E) in old birds ( $\geq 16$ years-old). Significant relationships are indicated with lines and confidence intervals around. When P-values were close to significant, dotted lines are indicated or the Pvalue written on the figure for boxplots. Males are shown in blue, while females are shown in red. 